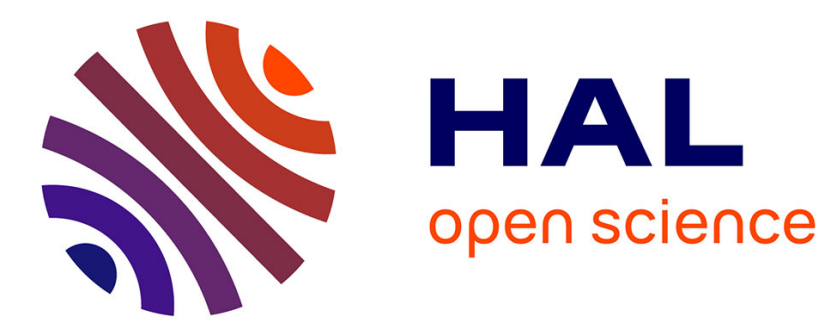

\title{
Measurement of acoustic velocity components in a turbulent flow using LDV and high-repetition rate PIV
}

Olivier Léon, Estelle Piot, Delphine Sebbane, Frank Simon

\section{To cite this version:}

Olivier Léon, Estelle Piot, Delphine Sebbane, Frank Simon. Measurement of acoustic velocity components in a turbulent flow using LDV and high-repetition rate PIV. Experiments in Fluids, 2017, 58

(6), pp.72. 10.1007/s00348-017-2348-4 . hal-01850568

\section{HAL Id: hal-01850568 \\ https://hal.science/hal-01850568}

Submitted on 2 Apr 2019

HAL is a multi-disciplinary open access archive for the deposit and dissemination of scientific research documents, whether they are published or not. The documents may come from teaching and research institutions in France or abroad, or from public or private research centers.
L'archive ouverte pluridisciplinaire HAL, est destinée au dépôt et à la diffusion de documents scientifiques de niveau recherche, publiés ou non, émanant des établissements d'enseignement et de recherche français ou étrangers, des laboratoires publics ou privés. 


\title{
Measurement of acoustic velocity components in a turbulent flow using LDV and high-repetition-rate PIV
}

\author{
Olivier Léon · Estelle Piot · Delphine Sebbane · Frank Simon
}

Received: date / Accepted: date

\begin{abstract}
The present study provides theoretical details and experimental validation results to the approach proposed by Minotti et al. (2008) for measuring amplitudes and phases of acoustic velocity components (AVC), that are waveform parameters of each component of velocity induced by an acoustic wave, in fully turbulent duct flows carrying multitone acoustic waves. Theoretical results support that the turbulence rejection method proposed, based on the estimation of cross-power spectra between velocity measurements and a reference signal such as a wall-pressure measurement, provides asymptotically efficient estimators with respect to the number of samples. Furthermore, it is shown that the estimator uncertainties can be simply estimated, accounting for the characteristics of the measured flow turbulence spectra. Two laser-based measurement campaigns were conducted in order to validate the acoustic velocity estimation approach and the uncertainty estimates derived. While in previous studies estimates were obtained using Laser Doppler Velocimetry (LDV), it is demonstrated that high-repetition-rate Particle Image Velocimetry (PIV) can also be successfully employed. The two measurement techniques provide very similar acoustic velocity amplitude and phase estimates for the cases investigated, that are of practical interest for acoustic liner studies. In a broader sense, this approach may be beneficial for non-intrusive sound emission studies in windtunnel testings.
\end{abstract}

Keywords Acoustic velocity measurement - duct flow · turbulence rejection

O. Léon $(\bowtie) \cdot$ E. Piot $\cdot$ D. Sebbane $\cdot$ F. Simon

ONERA, 2 Avenue Édouard Belin, 31055 Toulouse Cedex 4, France

E-mail: olivier.leon@onera.fr

\section{Introduction}

In a context of increasing efforts towards aircraft noise reduction from the aeronautical industry, measuring acoustic fields and localizing sound sources in a reliable manner in wind-tunnel tests are of major importance. While microphones are well suited for this task in quiescent environments, the use of pressure probes for in-flow acoustic measurements is rendered difficult for two main reasons. First, the flow turbulence usually interferes with the acoustic signal to be measured, requiring the use of tailored filtering and processing methods (Chung 1977). Second, such a measurement technique is intrusive, the probe usually perturbing the flow field, and may not be adequate when complex models or test section geometries are studied. Measuring wallpressure fluctuations may offer an appropriate way of evaluating acoustic fields and sound sources but it may be rendered difficult especially when the development of turbulent boundary layers have to be considered for accurate estimations.

In order to address these issues, various measurement techniques relying on optical methods have been proposed over the past decades, many of which are detailed for example by Valière (2014). As reported by Minotti et al. (2008), an approach based on Laser Doppler Velocimetry (LDV) for acoustic liner investigations was developed at ONERA in order to measure simultaneously acoustic velocity components (AVC), through a rejection of the flow turbulence, and turbulent flow velocities. Details regarding the technique itself will be provided in Sec. 2. Relying on LDV is suited for AVC estimation for two main reasons. First, the velocity dynamic range of an LDV system is high, usually greater than $10^{4}$, which can be of importance when studying acoustic waves at low sound pressure levels in a flow. For example, as also highlighted by Haufe et al. (2013), considering an acoustic wave at a sound pressure level of $120 \mathrm{~dB}$, giv- 
ing an acoustic velocity amplitude around $70 \mathrm{mms}^{-1}$, carried in a flow at a Mach number $M=0.2$, which gives a mean flow velocity around $70 \mathrm{~m} \mathrm{~s}^{-1}$ under normal temperature and pressure, yields a velocity dynamic range of about $10^{3}$. For a lower sound pressure level of $100 \mathrm{~dB}$, the velocity dynamic range to reach with the same flow is about $10^{4}$. Second, the acquisition rate can be greater than $20 \mathrm{kHz}$, allowing to perform AVC estimations in the spectral domain, over a large frequency bandwidth in the human hearing range. Using microphone measurements as a reference, Minotti et al. (2008) showed the consistency of the technique proposed to estimate AVC using LDV in a fully turbulent duct flow, with a bulk Mach number $M_{b}=0.1$, carrying traveling plane acoustic waves at a sound pressure level around $130 \mathrm{~dB}$ and a frequency of a few kilohertz. Further successful applications of this technique for acoustic liner studies at higher Mach numbers were later reported by Heuwinkel et al. (2010), Piot et al. (2010) and Betgen et al. (2012). It is worth noting however that relying on LDV, which provides single-point velocity measurements, to estimate AVC in a plane or a volume with a fine spatial resolution can be time-consuming and alternative techniques may be desirable.

While this technique has been employed for several years for acoustic liner investigations at ONERA, no detailed theoretical analysis of its domain of validity has been reported in the literature up to date. One objective of the present work is thus to provide further details on this acoustic velocity component estimation method, as well as to study the uncertainty associated with the estimators in order to assess its efficiency.

Also relying on the Doppler effect, a Doppler Global Velocimetry technique with frequency modulation (FM-DGV), initially developed by Fischer et al. (2007) and further detailed by Fischer et al. (2013), was applied by Haufe et al. (2012) and Haufe et al. (2013) for acoustic liner studies. The former authors showed the applicability of the technique to extract AVC yielded by single-tone acoustic excitations at sound pressure levels ranging from $120 \mathrm{~dB}$ to $130 \mathrm{~dB}$ in duct flows with Mach numbers up to 0.3. In the work presented by Haufe et al. (2013), a linear array of 23 fibercoupled avalanche photo detectors was employed to obtain a 2D measurement field of the three acoustic velocity components, which are obtained by repeating the measurement three times with three different observation directions. Similarly to LDV systems, this technique provides high acquisition rates and high velocity dynamic ranges. However, since a significant number of detectors may be used, this technique could be less time-consuming than LDV for acousticfield characterization.

Of particular interest, a different approach than the one proposed by Minotti et al. (2008) for AVC estimation has been developed by Haufe et al. (2012). Indeed, these au- thors showed that, relying only on Fourier transforms of the velocity measurements, one could build efficient estimators of acoustic velocity components. These estimators, however, are biased when a phase reference is unknown and this bias was shown to be inversely proportional to the square root of the measurement duration. One advantage, however, of this approach compared to the one previously presented is that no additional signal is required, which can be of great importance for some applications.

Among the other optical techniques that have been developed for acoustic measurement, an interesting approach relying on Rayleigh-scattering was investigated by Rausch et al. (2012). This proof-of-concept showed that Rayleighscattering could be used for direct acoustic density fluctuation measurements. These measurements, however, were performed without flow, thus without Mie-scattering issues yielded by dust particles for example. The application of this technique to liner studies could then be challenging due its sensitivity to parasitic light and vibrations.

Finally, another optical technique largely employed in the literature for acoustic measurement with a flow, and particularly for acoustic liner studies, is Particle Image Velocimetry (PIV). Among the studies relying on PIV for such applications, one may refer to the works of Fischer et al. (2008), Heuwinkel et al. (2010) and Alomar and Aurégan (2017). The advantages of this technique, compared to the ones previously presented, mainly lie in its ease of use and in the high image resolution that one can achieve, the measurements being performed in a plane using sensors that can have a resolution of several megapixels. One limitation, however, is its lower velocity dynamic range compared to Dopplerbased systems, since maximum values of about 400 are usually given. While this can limit the possible cases of study, it is emphasized that many practical cases of interest for acoustic liner research do not require higher velocity dynamic ranges.

To the knowledge of the authors, such PIV-based acoustic velocity measurements in turbulent flows have only been conducted using low-repetition-rate PIV systems that were phase-locked to a reference signal in order to perform acoustic velocity estimations using phase-averaging techniques. Nonetheless, it is worth noting that a few studies dealing with acoustic particle velocity measurements in quiescent environments have already been successfully employing highrepetition-rate PIV to evaluate acoustic velocity spectra, see for example the work of Henning et al. (2013). Relying on a phase-averaging approach is accurate but its ease of use highly depends on the ease of definition of phase classes from the reference signal employed. Consequently, in the works previously cited using a phase-averaging approach, only single-tone acoustic waves in duct flows were studied. When it comes to multi-tone acoustic waves, such an approach is rapidly limited whereas the techniques previously 
presented for AVC estimations from LDV and FM-DGV measurements are not, since they work in the frequency domain. A second objective of the present paper is thus to demonstrate the applicability of an acoustic velocity component estimation technique such as the one presented by Minotti et al. (2008) to high-repetition-rate PIV measurements, which would thus be suited for liner studies involving multi-sine acoustic excitations. Multi-sine acoustic excitation is of practical interest for acoustic liner investigations since it allows to study the response of a liner to various frequencies through only one measurement campaign, which is thus considerably time-saving. Furthermore, some liners have different impedance responses depending on the spectral content of the excitation (Bodén 2013), which can contain a large number of tones in a context of fan applications. It is thus desirable to provide adequate experimental tools for such studies.

The manuscript is organized as follows. Sec. 2 provides the theoretical details behind the AVC estimation method, deriving estimator uncertainties and discussing the effect of flow turbulence. Then, Sec. 3 describes the experimental facility and setups that were employed in order to investigate the application of the estimation method presented. A short description of the flow characteristics in which acoustic waves were introduced is given in Sec. 4. Finally, results of the AVC estimations from LDV and PIV measurements are presented in Sec. 5 and conclusions are drawn in Sec. 6.

\section{Estimation method of acoustic velocity components}

Following the work of Minotti et al. (2008), we aim at providing robust estimators of the RMS amplitudes and phase differences from a reference signal of acoustic velocity components (referred to as AVC in the following) when multisine acoustic waves are carried in a fully turbulent flow. This section details the theoretical background behind this AVC estimation method and presents the uncertainty associated with the statistical process.

\subsection{Motivation}

Assessing acoustic velocity components through flow velocity measurements in a fully turbulent flow is not straightforward. For example, Fig. 1 provides the power spectral density (PSD) of axial velocity measured by LDV in a fully turbulent duct flow, above an acoustic liner, at a bulk Mach number $M_{b}=0.2$ and where several acoustic waves were introduced at various frequencies. The sound pressure level of each downstream-traveling wave was set to $130 \mathrm{~dB}$. This figure highlights that, because the flow turbulence is not negligible at the frequencies of interest, acoustic velocity amplitudes associated with these acoustic waves cannot be di-

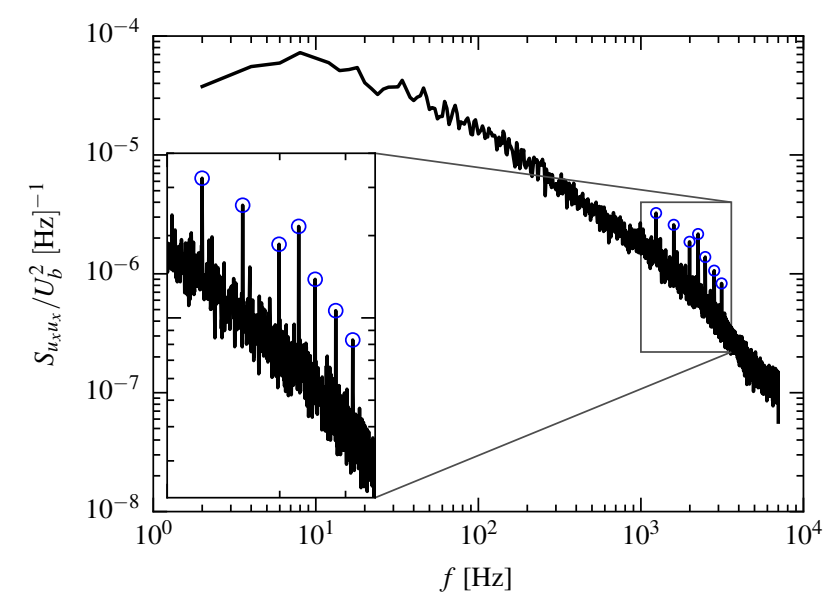

Fig. 1: Power spectral density (PSD) of dimensionless axial velocity $u_{x} / U_{b}$ measured by LDV in a fully turbulent duct flow $\left(M_{b}=0.2\right)$ with seven plane acoustic waves at SPLs equal to $130 \mathrm{~dB}$ and at various frequencies above $1 \mathrm{kHz}$; spectral peaks associated with these acoustic waves are highlighted using markers $(\mathrm{O})$.

rectly estimated from such spectra in an accurate manner. The spectral peaks observed also contain the contribution of measurement noise and flow turbulence, introducing a bias in such a direct estimation approach. Consequently, specific estimation techniques such as the one exposed in the following or the one proposed by Haufe et al. (2012) are required.

\subsection{Theoretical background}

The flow velocity fields $\mathbf{u}=\left\{u_{i}\right\}_{i \in \llbracket 1,3 \rrbracket}^{T}$ that we will consider can be decomposed into four parts, such that

$\mathbf{u}(\mathbf{x}, t)=\mathbf{u}_{0}(\mathbf{x})+\tilde{\mathbf{u}}(\mathbf{x}, t)+\mathbf{u}^{\prime}(\mathbf{x}, t)+\mathbf{b}(\mathbf{x}, t)$

where $\mathbf{u}_{0}$ is the mean flow field, $\tilde{\mathbf{u}}$ represents the acoustic velocity vector field, $\mathbf{u}^{\prime}$ gathers the turbulent fluctuations, and $\mathbf{b}$ is some measurement noise, inherent to the acquisition process. A component $i$ of the instantaneous velocity field generated by a multi-sine acoustic perturbation can be written in the following manner,

$\tilde{u}_{i}(\mathbf{x}, t)=\sum_{k=1}^{K} \sqrt{2} A_{\tilde{u}_{i}}\left(\mathbf{x}, \omega_{k}\right) \cos \left[\omega_{k} t+\phi_{\tilde{u}_{i} s}\left(\mathbf{x}, \omega_{k}\right)\right]$,

where $A_{\tilde{u}_{i}}\left(\mathbf{x}, \omega_{k}\right)$ is the expected acoustic velocity RMS amplitude at a frequency $\omega_{k}, \phi_{\tilde{u}_{i} s}\left(\mathbf{x}, \omega_{k}\right)$ the expected acoustic phase difference with respect to a reference signal $s(t)$ at a frequency $\omega_{k}$, and $K$ the total number of acoustic sine waves considered.

Within this formalism, we assume that the turbulent fluctuations $\mathbf{u}^{\prime}$ are not correlated with the acoustic velocity field 
$\tilde{\mathbf{u}}$. This is the case when the acoustic perturbations do not alter the flow dynamics and are simply carried by the flow, and when the turbulence itself does not produce any significant acoustic perturbation at the frequencies of interest. However, when acoustic perturbations and turbulence are coupled, the following approach will not separate the respective components. Interpretation of the results obtained in such cases should then be made with caution, as exemplified by Haufe et al. (2012).

We highlight nonetheless that extracting from a flow the turbulent velocity fluctuations that are coupled with the acoustics is also of interest for the aeroacoustic community. Such an objective was for example sought in the works of Henning et al. (2010) and Breakey et al. (2013), who performed simultaneous PIV and microphone measurements on different configurations. The present estimation method may then be also beneficial for such studies.

Under this assumption, we show in the following that the expected acoustic velocity RMS amplitudes $A_{\tilde{u}_{i}}$ and phase differences $\phi_{\tilde{u}_{i} s}$ can be obtained through the evaluation of the cross power spectral function $G_{u_{i} s}(\mathbf{x}, \omega)$ between the velocity component $u_{i}(\mathbf{x}, t)$ and a reference signal $s(t)$ directly linked to the acoustic perturbations we aim at measuring. This signal $s(t)$ is for example the input signal of the loudspeaker generating the sound carried by the flow, or an unsteady pressure signal measured on the wall of the duct, at a distance from the measurement volume typically greater than the turbulence integral length scales. It is worth noting that using such a reference signal simplifies the estimation process described in the following and, in particular, ensures that the estimators built are non-biased, which is not the case when a reference phase is not defined as detailed by Haufe et al. (2012). Indeed, as shown by these authors, relying solely on the Fourier transforms of the velocity signals renders the estimators biased, with a systematic deviation dependent on the number of samples and on the noise level.

In the present case, the cross power spectral function writes

$G_{u_{i} s}(\mathbf{x}, \omega)=G_{\tilde{u}_{i} s}(\mathbf{x}, \omega)+G_{u_{i}^{\prime} s}(\mathbf{x}, \omega)+G_{b_{i} s}(\mathbf{x}, \omega)$,

using the linearity of both the expectation function and the Fourier transform. Following the previous assumptions, it theoretically comes

$G_{u_{i}^{\prime} s}=0$

$G_{b_{i} s}=0$

and the expected acoustic velocity RMS amplitude and phase can then be evaluated at a desired frequency $\omega_{k}$ by

$\begin{aligned} A_{\tilde{u}_{i}}\left(\mathbf{x}, \omega_{k}\right) & =\frac{\left|G_{u_{i} s}\left(\mathbf{x}, \omega_{k}\right)\right|}{\sqrt{\left|G_{s s}\left(\omega_{k}\right)\right|}} \\ \phi_{\tilde{u}_{i} s}\left(\mathbf{x}, \omega_{k}\right) & =\left\langle G_{u_{i} s}\left(\mathbf{x}, \omega_{k}\right)\right\rangle\end{aligned}$ where $|$.$| refers to the norm and \langle$.$\rangle to the angle of a com-$ plex number.

The approach retained in the present study to obtain an estimator $\hat{G}_{u_{i} s}$ of the cross power spectral function $G_{u_{i} s}$ relies on a WOSA method (Welch's Overlapped Segmented Averaging method, also referred to as the Welch's method), which is a non-parametric asymptotically unbiased estimation method (Stoica et al. 2005). In this method, the measured data is split in overlapping segments of length $N_{F F T}$ that are windowed and Fourier transformed. To compute power spectra estimates, the squared magnitude of the results obtained with one signal is evaluated, providing a set of periodograms. The average of these periodograms provides a power spectrum, or a power spectral density (PSD) depending on the scaling, denoted as $S$ in the following. The estimation of a cross power spectrum between two signals follows the same procedure, but with the periodograms here obtained through a product of the two sets of Fourier transformed segments.

While the expressions provided in Eq. (5) are theoretically correct, the practical construction of the acoustic velocity RMS amplitude and phase estimators, respectively denoted as $\hat{A}_{\tilde{u}_{i}}$ and $\hat{\phi}_{\tilde{u}_{i} s}$, may be performed in a slightly different manner.

Indeed, for the acoustic velocity amplitude estimator $\hat{A}_{\tilde{u}_{i}}$, spectral leakage and amplitude errors yielded by the choice of a windowing function have to be taken into account for an accurate, unbiased estimation. To do so, a first approach can be to work with cross power spectra (CPS), accounting for the appropriate effective noise bandwidth (ENBW), which depends on the window function employed. The evaluation of $\hat{A}_{\tilde{u}_{i}}$ is then directly performed at the closest frequencies of interest $\omega_{k}$. The choice of window function is then of great importance, since it has to minimize the maximum amplitude error: usually, flat-top windows are considered, and we made the choice of the HFT70 window as developed by Heinzel et al. (2002), ensuring a maximum error of $0.075 \%$.

A second approach may be to work with cross power spectral densities (CPSD). Spectral leakage can then be accounted for by integrating $\left|\hat{G}_{u_{i} s}\right|$ on a frequency bandwidth around the desired frequency, thus such that

$\hat{A}_{\tilde{u}_{i}}\left(\mathbf{x}, \omega_{k}\right)=\frac{\int_{\omega_{k}-n \delta \omega}^{\omega_{k}+n \delta \omega}\left|\hat{G}_{u_{i} s}\left(\mathbf{x}, \omega_{j}\right)\right| d \omega_{j}}{s_{\mathrm{rms}}\left(\omega_{k}\right)}$

where $s_{\mathrm{rms}}$ refers to the RMS amplitude of the reference signal $s$ and $n \delta \omega$ typically covers 3 frequency bins using a Hanning window.

Regarding the acoustic phase difference estimator $\hat{\phi}_{\tilde{u}_{i} s}$, the simplest method is to evaluate the phase of $\hat{G}_{u_{i} s}$ at the closest desired frequency, on a single frequency bin. A second possible approach, that one might think to be more robust, can be to calculate the phase of the sum of the CPS 
around the closest frequency of interest $\omega_{k}$, which can be seen as a weighted average, such that

$\hat{\phi}_{\tilde{u}_{i} s}\left(\mathbf{x}, \omega_{k}\right)=\left\langle\frac{1}{2 n+1} \sum_{j=-n}^{n} \hat{G}_{u_{i} s}\left(\mathbf{x}, \omega_{k}+j \delta \omega\right)\right\rangle$

where $n$ would again cover a few adjacent frequency bins. Sec. 2.3 will highlight and conclude on the performances of these amplitude and phase difference estimation methods in the presence of synthetic noise.

Since the acoustic amplitude and phase estimations are performed in the frequency domain, the spectral richness of the acoustic velocity components is not a limiting factor as it would be using a phase-averaging approach. This holds as long as the Nyquist-Shannon theorem is satisfied and the acoustic frequencies are well separated in the power spectra estimates.

2.3 Efficiency and uncertainty of the estimation assuming a white Gaussian noise

In practice, since the number of samples $N$ is limited, the finite-time CPS estimator of $u_{i}$ with $s$, that is $\hat{G}_{u_{i} s}$, will actually depend on the flow turbulence and the measurement noise.

For the sake of simplicity, let us assume for now that the two random variables $u_{i}^{\prime}$ and $b_{i}$ can be modeled as a single white Gaussian noise (WGN) $\mathbf{w}=\mathscr{N}\left(0, \sigma_{w}^{2}\right)$. Furthermore, let us also consider a single sine wave, the following results being independent of the number $K$ of acoustic waves considered. Based on Kay (1984), the minimum achievable standard deviations in the presence of WGN for any estimation of $A_{\tilde{u}_{i}}$ and $\phi_{\tilde{u}_{i} \text { s }}$ defined in Eq. (2) are given by the respective Cramér-Rao lower bounds (CRLBs), which are expressed in the following manner,

$\sigma_{\hat{A}_{\tilde{u}_{i}}} \geq \frac{\sigma_{w}}{\sqrt{N}}$

$\sigma_{\hat{\phi}_{\tilde{u}_{i} s}} \geq \frac{1}{\sqrt{N}} \frac{\sigma_{w}}{A_{\tilde{u}_{i}}}$

where $N$ is the total number of samples. Providing the definition of the signal-to-noise ratio

$\mathrm{SNR}=A_{\tilde{u}_{i}}^{2} / \sigma_{w}^{2}$,

these bounds then write

$\frac{\sigma_{\hat{A}_{\tilde{u}_{i}}}}{A_{\tilde{u}_{i}}} \geq \frac{1}{\sqrt{N}} 10^{-\mathrm{SNR}[\mathrm{dB}] / 20}$,

$\sigma_{\hat{\phi}_{\tilde{u}_{i} s}} \geq \frac{1}{\sqrt{N}} 10^{-\mathrm{SNR}[\mathrm{dB}] / 20}$,

where $\mathrm{SNR}[\mathrm{dB}]=10 \log _{10} \mathrm{SNR}$.

In order to illustrate and to assess the efficiency of the present estimation method in comparison with these bounds,

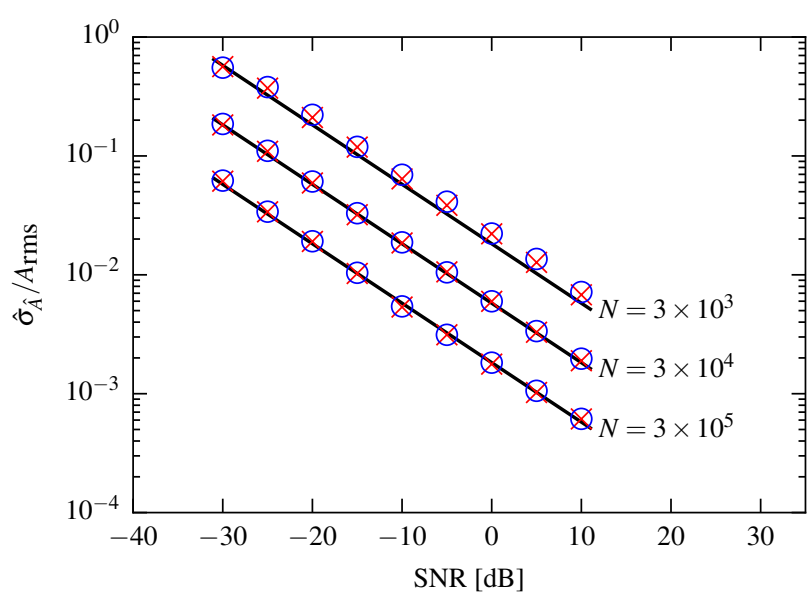

(a) the red crosses $(\times)$ are obtained from RMS amplitude estimations yielded by the ENBW-corrected CPS method; the blue circles $(\mathrm{O})$ are obtained from estimations based on the integrated-CPSD method given by Eq. (6).

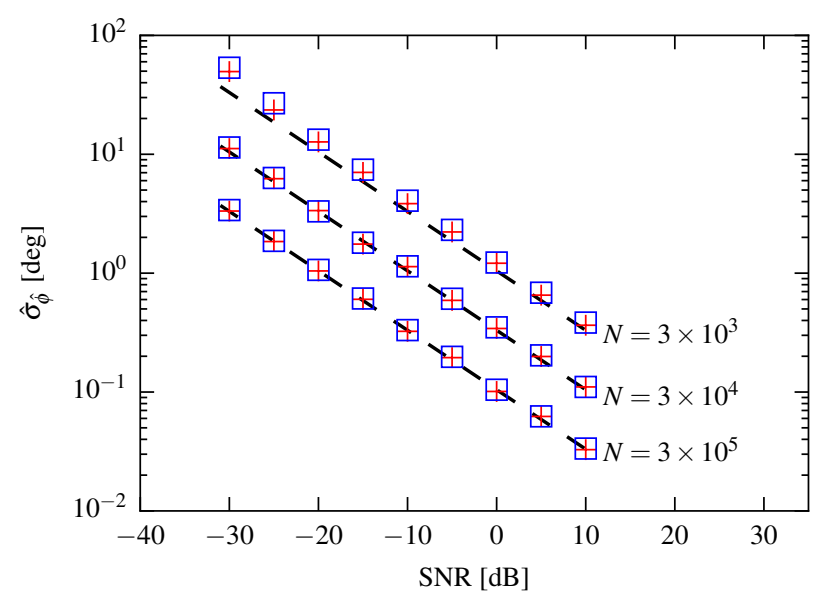

(b) the red plus symbols $(+)$ are obtained from phase estimations obtained on a single frequency bin; the blue squares $(\square)$ are obtained from estimations based on Eq. (7).

Fig. 2: Estimated standard deviation of (a) the RMS amplitude estimator $\hat{A}$ and (b) the phase difference estimator $\hat{\phi}$ for various levels of WGN in terms of signal-to-noise ratio (SNR) expressed in decibels; the solid and dashed black lines ( $\longrightarrow$ and - - - ) are the Cramér-Rao lower bounds, as provided by the bounds of the inequalities (10), obtained for three number of points $N$; estimated standard deviations $\hat{\sigma}$ are calculated using 500 independent simulations.

we consider the results obtained on a synthetic test case where the present simplifications are applied (WGN and single sine wave). The signal sampled at a frequency $f_{s}=1 / d t$ thus writes

$u\left(t_{n}\right)=\sqrt{2} A_{\mathrm{rms}} \cos \left(2 \pi f t_{n}+\phi\right)+w\left(t_{n}\right)$, 
where $f$ is the synthetic acoustic signal frequency, $t_{n}=n d t$ with $n \leq N, N$ being the total number of time samples, and $w(t)$ is the WGN. As long as the sampling theorem is satisfied, the values of $f$ and $f_{s}$ do not play any major role in the following, since WGN provides a uniform power spectrum. The reference signal is taken such that $s\left(t_{n}\right)=\cos \left(2 \pi f t_{n}\right)$. The CPS estimates $\hat{G}_{u s}$ are then calculated using the WOSA method with a window of size $N_{F F T}=1024$. Depending on the estimation method used, either the HFT70 or the Hanning window is employed, with respective optimal overlaps equal to $72.2 \%$ and $50 \%$. Based on these CPS estimates, RMS amplitude and phase estimates $\hat{A}$ and $\hat{\phi}$ can be computed relying of the methods provided in Sec. 2.2. It was then verified that the different estimators proposed were asymptotically unbiased. By repeating this process, here 500 times, standard deviation estimates of each estimator, that is $\hat{\sigma}_{\hat{A}}$ and $\hat{\sigma}_{\hat{\phi}}$, can be computed. The results of these standard deviation estimations obtained for three number of samples $N \in\left\{3 \times 10^{3}, 3 \times 10^{4}, 3 \times 10^{5}\right\}$ are gathered in Fig. 2. In this figure are also provided the corresponding CRLBs as obtained considering the lower bounds of the inequalities (10). While for the lowest value of $N$ the uncertainties associated with the RMS amplitude and phase difference estimators tend to be slightly greater than their respective CRLBs, higher values of $N$ lead to a satisfactory correspondence over a large range of SNR. The ranges of SNR and number of samples $N$ here considered being fully representative of the ones found in the experiments conducted in the present study, this analysis supports the relevance of the estimation method for practical applications.

These results also support that in the presence of WGN, the RMS amplitude and phase difference estimation method proposed is asymptotically efficient, the estimators being asymptotically unbiased and their variance converging to the CRLBs. To support this assertion, Fig. 3 shows the convergence of the estimated standard deviations of these estimators to the Cramér-Rao lower bounds with an increasing number of samples $N$ considered, for the case of a signal-tonoise ratio of $-20 \mathrm{~dB}$. For this case of study, about $3 \times 10^{4}$ samples are necessary to reach these lower bounds with an difference relative to the expected value lower than $2 \%$. It will be shown in Sec. 5.2 that such an asymptotic behavior is also observed with experimental data.

Furthermore, it can be observed in Fig. 2 and Fig. 3 that the different methods proposed in Sec. 2.2 to estimate RMS amplitudes and phase differences yield similar results. Nonetheless, slightly better estimates in terms of uncertainty are obtained for the amplitude using the ENBW-corrected CPS method, and for the phase difference using the single frequency bin method. Consequently, in the following, only these two velocity component estimation techniques will be considered.

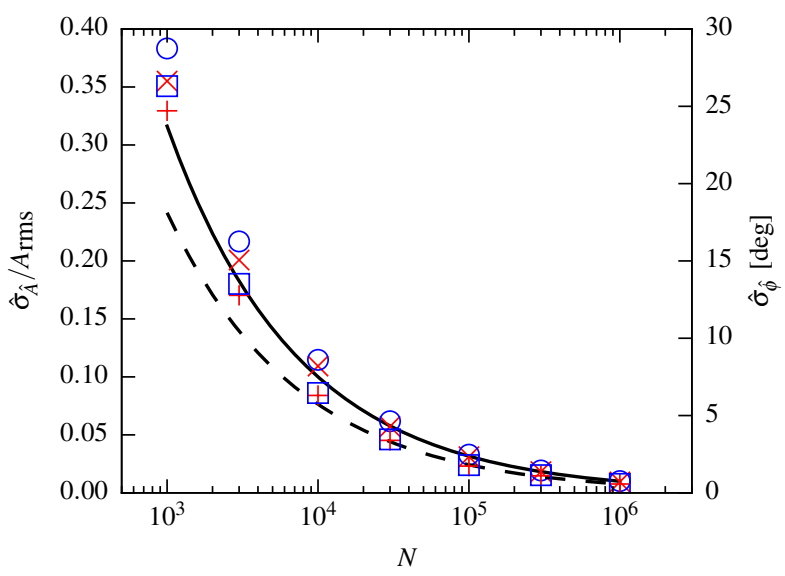

Fig. 3: Estimated standard deviation $\hat{\sigma}$ of the RMS amplitude and phase difference estimators $\hat{A}$ and $\hat{\phi}$ as a function of the number of points $N$ considered for a signal to noise ratio $\mathrm{SNR}=-20 \mathrm{~dB}$; see the legends of Fig. 2 for the definition of the markers; the solid and dashed black lines and - - ) are respectively the amplitude and the phase estimator CRLBs as obtained considering the inequalities (10).

\subsection{On the effect of colored noise on the estimation uncertainty}

White Gaussian noise employed in the previous synthetic model to simulate the effect of measurement noise and turbulence in the estimation process provides a uniform power spectral density as displayed in Fig. 4 with the black solid line. This renders the previous analysis frequency-independent and allowed to compare the results with the theoretical CRLBs. However, as also shown in this figure, the actual power spectral density of the turbulence, obtained from measurements presented in the following Sec. 4 and displayed using a red solid line, features a classical negative slope in log scales, here estimated around -0.7 for frequencies ranging from $10^{2} \mathrm{~Hz}$ to $10^{3} \mathrm{~Hz}$. We emphasize that the measured PSD and the synthetic one relying on WGN have the same power $\sigma_{w}^{2}$. From this figure, because of such a non-uniform distribution of power spectral density, one can thus expect that the actual uncertainties of the acoustic velocity component estimates will be frequency-dependent.

In order to quantitatively explore this point and to provide a way to assess more accurately the uncertainties for the following experimental part, we modify our previous model by replacing the WGN by a colored noise with appropriate parameters in order to display a spectral slope in log scales similar to the one measured.

The estimated PSD obtained using this model, with a power $\sigma_{w}^{2}$ equal to the measured one, is shown in Fig. 4 using a blue solid line. Clearly, regarding the power spectral 


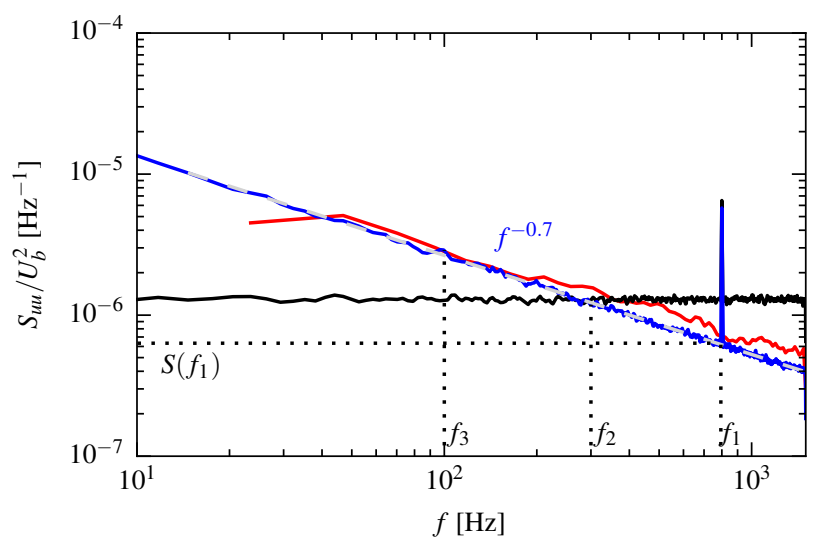

Fig. 4: Estimated power spectral density $S_{u u}(f) / U_{b}^{2}$ of three signals; _ axial velocity $u_{x}$ measured by PIV on the duct axis in Sec. 4 obtained using $N=3 \times 10^{4}$ samples and a sampling frequency $f_{s}=3 \mathrm{kHz}$; — made of WGN, with variance $\sigma_{w}^{2}$ equal to that of the measured PIV signal, and a $f_{1}=800 \mathrm{~Hz}$ tonal component representative of an acoustic wave with a SPL of $130 \mathrm{~dB}$ as studied in Sec. 5 (SNR = -19.2 dB); - synthetic signal made of colored noise with a spectral exponent of -0.7 , with variance equal to $\sigma_{w}^{2}$, and the same tonal component as the one in the previous synthetic signal.

density, this model is more representative of the measurements. In particular, the case here depicted aims at studying an acoustic wave at a frequency $f_{1}=800 \mathrm{~Hz}$, at a sound pressure level $L=130 \mathrm{~dB}$, in a fully turbulent duct flow yielding a SNR of $-19.2 \mathrm{~dB}$, with $N=3 \times 10^{4}$ samples and an acquisition frequency $f_{s}=3 \mathrm{kHz}$, which are the PIV experimental parameters found in Sec. 5 .

Similarly to the analysis conducted in Sec. 2.3 with a model relying on WGN, we conducted an analysis on the standard deviations of the RMS amplitude and phase difference estimators with this modified model. Because of the frequency-dependence of this model, for the sake of clarity, we only provide here results obtained with one number of samples $N=3 \times 10^{4}$, since the conclusions drawn are largely independent of $N$. Fig. 5 shows the estimated standard deviations of the estimators as a function of the SNR for the three frequencies defined in Fig. 4. It can be observed that the estimations performed at a frequency $f_{2}=300 \mathrm{~Hz}$, thus where the model based on WGN and the one using colored noise display an equivalent PSD level in Fig. 4, lead to similar uncertainty levels, as one could expect. However, both the amplitude and the phase estimations performed at lower frequencies such as $f_{3}=100 \mathrm{~Hz}$ present higher uncertainty levels compared to the ones provided by the synthetic model relying on WGN. In an opposite manner, estimations performed at higher frequencies, such as $f_{3}=800 \mathrm{~Hz}-$ the

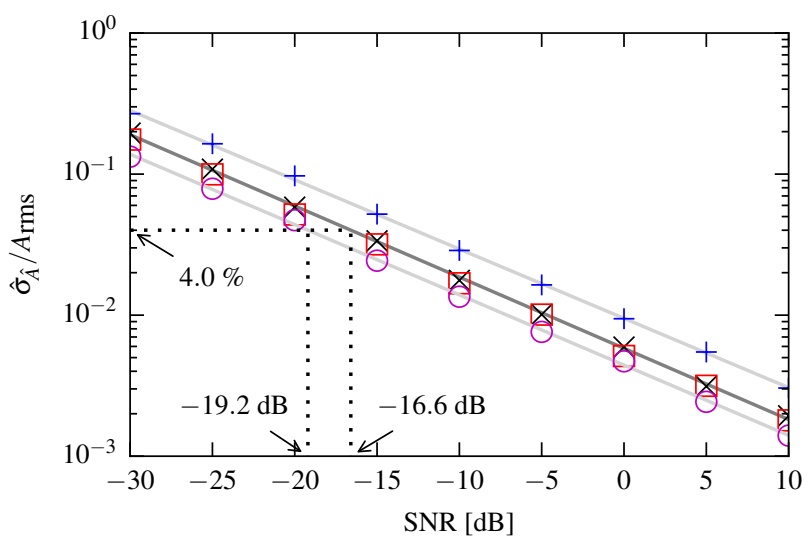

(a)

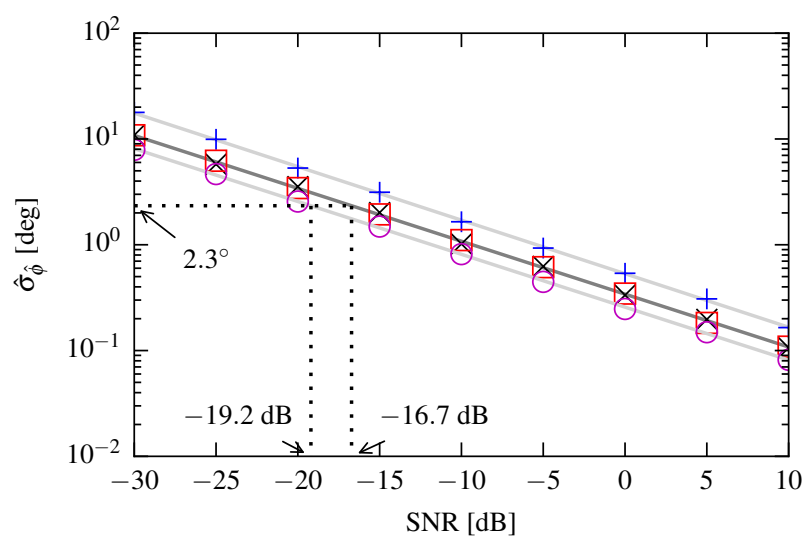

(b)

Fig. 5: Estimated standard deviation of (a) the RMS amplitude and (b) the phase difference estimators as a function of SNR in decibels, for a number of samples $N=3 \times 10^{4}$, obtained with a synthetic model using colored noise representative of the spectral behaviour of the turbulence measured in the following experiments (see Fig. 4). Results obtained for various frequencies; $\bigcirc: f_{1}=800 \mathrm{~Hz} ; \square: f_{2}=300 \mathrm{~Hz}$; + : $f_{3}=100 \mathrm{~Hz}$. Results previously obtained with a synthetic signal made of WGN are displayed with the symbol $\times($ see Fig. 3). Grey solid lines represent linear interpolations. Standard deviations are estimated using 500 independent simulations.

experimental acoustic frequency in Sec. 5 -, present lower uncertainty levels. More precisely, considering this last practical case of study with a SNR of $-19.2 \mathrm{~dB}$, corresponding to a sound pressure level of $130 \mathrm{~dB}$, the estimated standard deviation of the amplitude estimator yielded using WGN is $5.4 \%$ of $A_{\mathrm{rms}}$, while the one obtained using colored noise is of $4.0 \%$ of $A_{\text {rms }}$. Regarding the estimated standard deviation of the phase difference estimator, it drops from $3.1^{\circ}$ down to $2.3^{\circ}$. 
Clearly, the spectral characteristics of the noise, and thus certainly of the turbulence when considering experimental data, is thus not negligible for such a study. Consequently, in the following experimental parts, reliable uncertainty estimates of AVC estimators should not be expected by solely relying on the frequency-independent signal-to-noise ratio as defined by eq. (9). In a practical manner, these results show that the higher the acoustic frequency, the lower the estimator uncertainties because of a decrease in the spectral variance of the turbulence. This effect is expected to be greater in the inertial range of the turbulence spectrum where the spectral exponent is significantly lower and close to $-5 / 3$, as shown in Sec. 4 , providing significant decreases in spectral power as the frequency is increased.

In order to be able to correctly quantify the uncertainty levels using the previous analysis conducted in Sec. 2.3, which is frequency-independent, it appears sufficient with the present synthetic model to evaluate a specific SNR at the frequency of interest, referred to as $\mathrm{SNR}_{f}$ in the following, for which the correct standard deviation for both the amplitude and the phase difference estimators at the frequency of interest are obtained with the model based on WGN. This is illustrated in Fig. 5: the correct standard deviation of the amplitude estimator for a SNR equal to $-19.2 \mathrm{~dB}$, which is equal to $4.0 \%$ of $A_{\mathrm{rms}}$, is obtained for a specific frequencydependent $\mathrm{SNR}_{f}$ of $-16.6 \mathrm{~dB}$ relying on the WGN model. A very close value of $\mathrm{SNR}_{f}$ is obtained for the phase difference estimator.

In the present case, a direct evaluation of this $\mathrm{SNR}_{f}$ can be obtained by considering an equivalent WGN with a PSD level equal to the one actually measured at the frequency of interest without acoustic excitation. For example, as highlighted in Fig. 4 for the frequency $f_{1}$, one can employ the PSD level $S\left(f_{1}\right) / U_{b}^{2}=6.3 \times 10^{-7} \mathrm{~Hz}^{-1}$ to evaluate the variance $\sigma_{w e q}^{2}$ of an equivalent WGN on a frequency bandwidth $f_{s} / 2$, where $f_{s}$ is the sampling frequency, such that

$\sigma_{w e q}^{2}=S\left(f_{1}\right) \frac{f_{s}}{2}$

which then yields the frequency-dependent signal-to-noise ratio in decibel

$\mathrm{SNR}_{f_{1}}[\mathrm{~dB}]=10 \log _{10}\left(\frac{A_{\mathrm{rms}}^{2}}{\sigma_{w e q}^{2}}\right)$.

In the present case we obtain a value of $-16.6 \mathrm{~dB}$, consistent with the one obtained from Fig. 5. Experimental results supporting this discussion regarding the effect of the spectra characteristics on the estimate uncertainties are presented in Sec. 5.

As a final comment, this analysis thus has a practical application since it may allow one to easily estimate the theoretical statistical uncertainty bounds of the estimation method for experimental cases of study, assuming that the effects of turbulence can be taken into account in such a way. Indeed, given the flow conditions, the acoustic frequency of study $f_{a}$ and an estimation of the power spectral density $S\left(f_{a}\right)$ measured on the base flow, one can use eq. (13) to evaluate $\mathrm{SNR}_{f_{a}}$ and then rely on the CRLBs as defined by the inequalities (10) to estimate such bounds.

We can emphasize however that relying on the CRLBs for such uncertainty level estimations of the acoustic velocity amplitudes and phase differences is only expected to be justified when acoustic waves do not modify the turbulence of the flow and are only carried by a base flow, as assumed in Sec. 2.2. Indeed, in such cases, the turbulence may be seen as some random noise and we may base our analysis on the value of $\mathrm{SNR}_{f}$. In cases where acoustic waves introduced and flow turbulence get coupled, the CRLBs as evaluated using this approach should no longer be valid due to modifications of the turbulence itself, part of which will not be seen as random noise. Nonetheless, as stated in Sec. 2.2, it does not preclude the application of the estimation method itself on such cases.

\section{Experimental setups}

The following sections are now devoted to the practical application of this estimation method. While there is no particular hindrance for its application to cases with multi-sine acoustic waves, for the sake of clarity we solely focus here on the case of a single sine acoustic plane wave carried by a fully turbulent duct flow. Furthermore, we focus our analysis on a duct simply composed of rigid walls. The objective is to keep the configuration simple in order to assess the validity of the approach by comparing LDV, high-repetition-rate PIV and wall-mounted microphone measurements. Examples of successful AVC estimations based on LDV measurements for liner studies with multi-sine acoustic waves were previously reported by Minotti et al. (2008) and Betgen et al. (2012).

\subsection{Aeroacoustic facility and flow conditions}

The experiments were conducted on the aero-thermo-acoustics bench of ONERA Toulouse, France. This bench was primarily designed to study the impedance of material samples such as acoustic liners through pressure measurements and laser velocimetry. A schematic of this facility is provided in Fig. 6.

The bench is about 4-meters long and air can be supplied with regulation in both temperature and mass-flow-rate. A square duct with cross-sectional dimensions of $50 \mathrm{~mm} \times 50 \mathrm{~mm}$ $(2 h \times 2 h)$ follows a flow-conditioner module and two loudspeakers mounted in pressurized chambers that provide acoustic excitations to the duct flow. These two loudspeakers are 


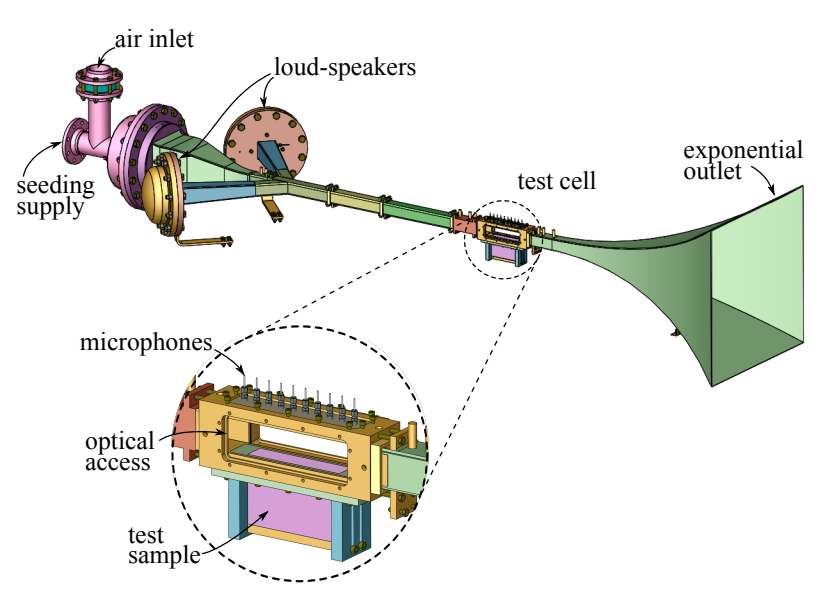

Fig. 6: Model of the aero-thermo-acoustic bench at ONERA Toulouse.

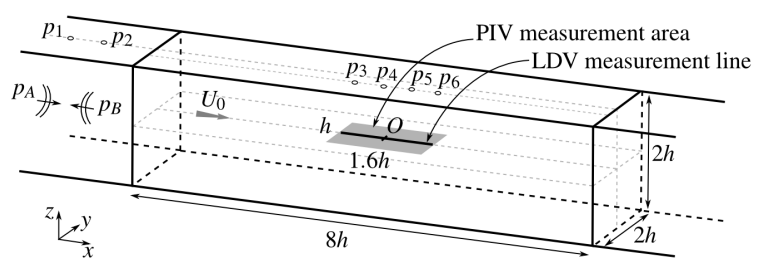

Fig. 7: Schematic of the test cell highlighting the dimensions and the measurement locations.

placed symmetrically on each side of the duct and are designed to generate plane acoustic waves in the test section. Consequently, in the following, only the axial acoustic velocity component will be studied. It was verified that, in the conditions of the present experiments, the other acoustic velocity components were negligible in the region of interest. The test cell, located $1340 \mathrm{~mm}$ (about 54h) downstream of the end of the contraction, is $200 \mathrm{~mm}$ long (8h) and is designed to be equipped on its sides with windows, pressure transducers and material samples such as acoustic liners. The duct termination is made of an exponential outlet connected to a muffled chamber in order to approach anechoic conditions. A schematic view of the test cell is shown in Fig. 7. As depicted in this figure, the direct reference frame $O x y z$ is defined such that the $x$-axis is aligned with the duct centerline, oriented streamwise, the $z$-axis is vertical oriented upward and the origin is taken as the geometrical center of the test section.

The present experiments were performed under normal atmospheric conditions, with the flow temperature regulated at the ambient temperature $T_{0}=20^{\circ} \mathrm{C}$ with maximum variations of $\pm 1^{\circ} \mathrm{C}$, yielding a speed of sound of $c_{0}=343 \mathrm{~m} \mathrm{~s}^{-1}$. The mass-flow-rate was set to $Q=100 \mathrm{~g} \mathrm{~s}^{-1}$, corresponding to a bulk velocity $U_{b}=33.2 \mathrm{~m} \mathrm{~s}^{-1}$ and a bulk Mach number $M_{b}=0.1$. The Reynolds number based on the bulk veloc-

\begin{tabular}{|c|c|c|c|c|c|c|}
\hline $\begin{array}{c}L \\
{[\mathrm{~dB}]}\end{array}$ & $\begin{array}{c}|\tilde{p}|_{\text {rms }} \\
{[\mathrm{Pa}]}\end{array}$ & $\begin{array}{l}|\tilde{u}|_{\mathrm{rms}} \\
{[\mathrm{m} / \mathrm{s}]}\end{array}$ & DVR & $\begin{array}{l}\text { SNR } \\
{[\mathrm{dB}]}\end{array}$ & $\begin{array}{c}\text { PIV } \\
\text { SNR }_{f} \\
{[\mathrm{~dB}]}\end{array}$ & $\begin{array}{l}\mathrm{LDV} \\
\mathrm{SNR}_{f} \\
{[\mathrm{~dB}]}\end{array}$ \\
\hline 130.5 & 67.0 & 0.16 & 176 & -19.2 & -16.1 & -22.5 \\
\hline 138 & 158.9 & 0.38 & 74 & -11.7 & -8.6 & -15 \\
\hline 145 & 355.7 & 0.86 & 33 & -4.7 & -1.5 & -7.9 \\
\hline 150 & 632.5 & 1.53 & 18 & 0.3 & 3.5 & -2.9 \\
\hline
\end{tabular}

Table 1: Sound pressure levels $L$ of the acoustic excitations introduced in the duct flow at a frequency $f_{a}=800 \mathrm{~Hz}$, together with estimates of the corresponding pressure and acoustic velocity RMS-amplitudes, dynamic velocity ranges (DVR) with the present flow conditions, signal-to-noise ratios (SNRs) assuming a turbulence rate of $4.4 \%$, and specific $\mathrm{SNR}_{f}$ estimated at the frequency $f_{a}$, based on the analysis performed in Sec. 2.4 with the adequate sampling frequencies $f_{s}$ and the values of $S_{u_{x} u_{x}}\left(f_{a}\right)$ obtained from Fig. 10.

\begin{tabular}{|c||c|c|c||c|c|c|}
\hline \multicolumn{1}{|c||}{} & \multicolumn{3}{c||}{ PIV } & \multicolumn{3}{c|}{ LDV } \\
\hline$L$ & $N$ & $\begin{array}{c}\hat{\sigma}_{\hat{A}} / \\
A_{\mathrm{rms}}\end{array}$ & $\hat{\sigma}_{\hat{\phi}}$ & $N$ & $\hat{\sigma}_{\hat{A}} /$ & $\hat{\sigma}_{\hat{\phi}}$ \\
{$[\mathrm{dB}]$} & & & & $A_{\mathrm{rms}}$ & \\
\hline 130.5 & 33,300 & $3.5 \%$ & $2.0^{\circ}$ & $2.5 \times 10^{5}$ & $2.5 \%$ & $1.4^{\circ}$ \\
138 & 22,200 & $1.8 \%$ & $1.0^{\circ}$ & $2.5 \times 10^{5}$ & $1.1 \%$ & $0.6^{\circ}$ \\
145 & 22,200 & $0.8 \%$ & $0.5^{\circ}$ & $2.5 \times 10^{5}$ & $0.5 \%$ & $0.2^{\circ}$ \\
150 & 22,200 & $0.5 \%$ & $0.3^{\circ}$ & $2.5 \times 10^{5}$ & $0.3 \%$ & $0.1^{\circ}$ \\
\hline
\end{tabular}

Table 2: Estimates of the uncertainty lower bounds of the acoustic velocity component estimators obtained with the experimental parameters of the PIV and the LDV measurements, based on the analysis of Sec. 2.4 and on the specific $\mathrm{SNR}_{f}$ provided in Tab. 1.

ity $U_{b}$ and the duct hydraulic diameter $d_{h}=2 h$ is $R e_{d_{h}}=$ $U_{b} d_{h} / v=1.06 \times 10^{5}$, ensuring the turbulent state of the flow.

Seeding for both LDV and PIV measurements was performed using fumed silica particles injected upstream of a flow rectifier and the convergent module, in order to ensure an homogeneous distribution. This choice of particle seeding over paraffin- or oil-based seeding is initially motivated by its weak deposit over time on the test cell windows. According to the manufacturer, the mean primary particle size is about $20 \mathrm{~nm}$, with aggregates up to $0.2 \mu \mathrm{m}$. These seed particles were observed to yield weak but sufficient Mie scattering for PIV measurements. Furthermore, considering the largest particles with a conservative maximum diameter of $0.5 \mu \mathrm{m}$, the minimum frequency response in our test conditions was estimated to be around $15 \mathrm{kHz}$ with less than $1 \%$ slip, and the maximum Stokes number to be around 0.025 , lower than 0.1 . These extremal values thus indicate that these seeding particles will not introduce any significant measurement bias due to inertia or drag. Finally, it was verified that the seeding introduced in the flow was not modifying in a significant manner the acoustic properties of the flow. 
Acoustic excitations were performed at a frequency $f_{a}=$ $800 \mathrm{~Hz}$ and with various sound pressure levels $L$ provided in Tab. 1. These sound pressure levels are defined such that

$L=20 \log _{10} \frac{|\tilde{p}|_{\mathrm{rms}}}{p_{\mathrm{ref}}}$,

where $p_{\text {ref }}=2 \times 10^{-5} \mathrm{~Pa}$ and $|\tilde{p}|_{\text {rms }}$ corresponds to the quadratic norm of pressure fluctuations induced by the downstreamtraveling acoustic wave. The values of the latter are also provided in Tab. 1 for completeness, together with estimates of the associated acoustic velocity RMS-amplitudes obtained assuming a uniform flow field, thus such that

$|\tilde{u}|_{\mathrm{rms}}=\frac{c_{0}}{\gamma} \frac{|\tilde{p}|_{\mathrm{rms}}}{p_{0}}$.

Estimates of the dynamic velocity ranges (DVR) can then be obtained considering the ratio of the duct flow bulk velocity $U_{b}$ with these acoustic velocity amplitudes. As observed in Tab. 1, these dynamic velocity ranges are well within the reach of both PIV and LDV measurement techniques for the sound pressure levels here considered. The difficulty of these measurements, however, lies in the low signal-to-noise ratio, as emphasized in Sec. 2. Here, the signal-to-noise ratio is defined as SNR $=|\tilde{u}|_{\text {rms }}^{2} /\left|u^{\prime}\right|_{\text {rms }}^{2}$, where $\left|u^{\prime}\right|_{\text {rms }}$ is the turbulence RMS amplitude. Considering a turbulence rate of $4.4 \%$ representative of the lowest turbulence intensity measured in Sec. 4 on the duct centerline, thus providing a turbulence RMS-amplitude of about $1.5 \mathrm{~m} \mathrm{~s}^{-1}$, the SNR obtained for each sound pressure level are given in Tab. 1. In addition, following the discussion of Sec. 2.4, the table also provides the more representative frequency-dependent signalto-noise ratio $\mathrm{SNR}_{f}$ estimated at the frequency $f_{a}=800 \mathrm{~Hz}$ for both PIV and LDV measurements using the PSD levels measured and reported in Sec. 4. Typically, measuring acoustic velocity components of waves at $130 \mathrm{~dB}$ with the present flow characteristics and with the present setups requires to deal at best with global signal-to-noise ratios of about $-19 \mathrm{~dB}$ since the turbulence increases towards the walls.

Based on our experience, we estimate that flush-mounted microphone measurements as performed in the present test conditions have a total uncertainty of about $0.3 \mathrm{~dB}$ at the sound pressure levels and frequencies here explored - a value that is approximate and that will serve as a comparative basis in the following sections. This is mainly the result of biases introduced by improper alignment of the probe with the test-cell wall. For the lowest SPL studied, this corresponds approximately to an uncertainty of $2 \%$ on the acoustic velocity amplitude. Regarding the acoustic phase, we usually consider a measurement uncertainty of $1^{\circ}$. Such uncertainties provide $95 \%$ confidence intervals of about $\pm 0.6 \mathrm{~dB}$ and $\pm 2^{\circ}$. Based on the theoretical results obtained in Sec. 2 , it is shown in Tab. 2 that, with the number of samples $N$ that were acquired through PIV and LDV measurements, satisfactory uncertainties on the acoustic velocity component estimates can be expected in comparison with these values for every SPL investigated here, except for the lowest one studied using PIV. Yet, as developed in Sec. 5.1, spatial information may be used to decrease the uncertainty of such a spectral estimate.

\subsection{Pressure measurements}

As the duct termination is not perfectly anechoic, downstream and upstream traveling plane acoustic waves, respectively referred to as $p_{A}$ and $p_{B}$ in Fig. 7, are found in the duct upon acoustic excitation by the loudspeaker modules. The respective amplitudes of these two waves were determined, as described in the following, through wall-pressure measurements labeled $p_{1}$ and $p_{2}$ in the same figure. These measurements were performed using two flush-mounted B\&K 4182 microphone probes, located $130 \mathrm{~mm}$ upstream of the test section at axial locations $x_{1}$ and $x_{2}$, and placed $28 \mathrm{~mm}$ apart. Following Bodén and Abom (1986) this separation distance is suitable to apply a two-microphones method (Chung and Blaser 1980) in a frequency range from $600 \mathrm{~Hz}$ to $3500 \mathrm{~Hz}$, the cutoff frequency of the bench. Microphone calibrations were performed using a $1 \mathrm{kHz}$ frequency calibrator B\&K 4231 and a correction to the microphone transfer function was applied in order to account for the thin tube behind which the microphone is mounted. Acquisitions were performed at a sampling frequency of $12.8 \mathrm{kHz}$ using a NI acquisition system (cDAQ-9178 with a NI 9234 module) over $3.7 \mathrm{~s}$, thus over about 3,000 acoustic periods.

Given the wall-pressure measurements $p_{1}$ and $p_{2}$, we thus aim at estimating the amplitudes of $p_{A}$ and $p_{B}$, as well as providing estimates of the acoustic velocity amplitudes and phases in the test cell for each acoustic excitation considered in Tab. 1 for validation of the laser-based estimates. Employing the complex notation and following Bodén and Abom (1986), the acoustic pressure can be written as $p(x, t)=$ $\Re\left(\hat{p}(x) e^{i \omega t}\right)$, where $\hat{p}(x)=A e^{-i k^{+} x}+B e^{-i k^{-} x}$ with $A$ and $B$ being the complex amplitudes and $k^{+}$and $k^{-}$the wavenumbers of respectively the downstream and the upstream traveling waves, providing the following linear system of equations in matrix-form at the frequency $\omega$,

$$
\left[\begin{array}{l}
\hat{p}_{1} \\
\hat{p}_{2}
\end{array}\right]=\left[\begin{array}{ll}
e^{-i k^{+} x_{1}} & e^{-i k^{-} x_{1}} \\
e^{-i k^{+} x_{2}} & e^{-i k^{-} x_{2}}
\end{array}\right]\left[\begin{array}{l}
A \\
B
\end{array}\right] .
$$

Assuming a uniform flow, the wavenumbers are given by

$k^{ \pm}=\frac{k}{M \pm 1}$

with $k=\omega / c_{0}$ and $M$ the flow Mach number, approximated in our case with $M_{b}$. The solution to Eq. (16) providing the 
complex amplitudes of the downstream and upstream waves writes

$A=\hat{p}_{1} \frac{\exp \left(\frac{i k x_{2}}{1-M}\right)-H_{21} \exp \left(\frac{i k x_{2}}{1-M}\right)}{2 i \sin \left(\frac{k \Delta x}{1-M^{2}}\right) \exp \left(\frac{i k M\left(x_{1}+x_{2}\right)}{1-M^{2}}\right)}$,

$B=-\hat{p}_{1} \frac{\exp \left(\frac{-i k x_{2}}{1+M}\right)+H_{21} \exp \left(\frac{-i k x_{1}}{1+M}\right)}{2 i \sin \left(\frac{k \Delta x}{1-M^{2}}\right) \exp \left(\frac{i k M\left(x_{1}+x_{2}\right)}{1-M^{2}}\right)}$,

with $\Delta x=x_{2}-x_{1}$ and $H_{21}=\hat{p}_{2} / \hat{p}_{1}$ the transfer function between the two microphones. This transfer function is estimated from simultaneous microphone measurements, and is further refined by alternatively switching the two microphones and calculating the mean transfer function, allowing to get rid of the intrinsic phase-shift of each microphone. Then, knowing $\hat{p}_{1}$ and $H_{21}$ for each test case, the respective amplitudes of the two traveling acoustic waves can be determined using Eq. (18). The sound pressure levels $L$ given in Tab. 1 are calculated based on $|A|$, the amplitude of the downstream-traveling wave.

Furthermore, still considering a uniform flow, the theoretical complex acoustic velocity writes

$\hat{u}(x)=\frac{1}{\rho_{0} c_{0}}\left(A e^{-i k^{+} x}-B e^{-i k^{-} x}\right)$,

allowing to estimate the acoustic velocity phases and amplitudes in the test cell at the axial locations where laser measurements were performed. We highlight that the acoustic velocity phases then obtained are phase differences with respect to the measurement $p_{1}$, while laser velocimetry systems introduce their own phase-shifts resulting from electronic delays. Furthermore, the absolute amplitudes obtained from this model directly depend on the amplitude of $\hat{p}_{1}$ and $H_{21}$, which are typically measured with an uncertainty of $0.3 \mathrm{~dB}$. Consequently, these amplitudes and phase-differences as obtained from Eq. (19) will not be considered as they are but will be slightly corrected in Sec. 5 using a simple nearunity factor on the amplitudes and a phase-shift on the phase differences. The corrected results will then be used to assess the validity of the axial trends of the laser-based estimates obtained.

In order to further validate the laser-based acoustic velocity estimates, direct wall-pressure measurements at four axial locations within the axial bounds of the PIV measurement area were performed for each test case presented in Tab. 1. These measurements were performed on the upper wall of the test cell at $z=h$, on a longitudinal line slightly off-axis at $y=-0.16 h=-4 \mathrm{~mm}$, at the axial positions $x / h \in$ $\{-0.72,-0.24,0.24,0.72\}$ labeled $p_{i}$ with $i \in \llbracket 3,6 \rrbracket$ in Fig. 7 . These pressure taps are located $12 \mathrm{~mm}$ apart. One flushmounted B\&K 4182 microphone was employed for these wall-pressure measurements, using the same acquisition parameters as the ones selected for $p_{1}$ and $p_{2}$. These measure- ments provide direct estimates of the acoustic pressure amplitudes and phase differences with respect to a reference signal. Estimates of the axial acoustic velocity amplitudes and phases are then deduced using Eq. (15), thus assuming a uniform flow field. It is emphasized that such an assumption, even if largely employed in the literature, can be questioned for the present duct flow application. Indeed, all refraction effects induced by the shear flow are then neglected, which may bias the microphone-based acoustic velocity estimations.

\subsection{Laser Doppler Velocimetry}

A two-component LDV system mounted on a 3-axis traverse system was used in forward-scattering configuration to maximize signal to noise ratios and data rates. Mean data rates obtained were typically greater than $15 \mathrm{kHz}$. This measurement system was composed of a $1 \mathrm{~W}$ argon-ion SpectraPhysics laser emitting green $(514.5 \mathrm{~nm})$ and blue $(488 \mathrm{~nm})$ wavelengths. The two pairs of beams were issued by a TSI9832 emitting head equipped with a $240 \mathrm{~mm}$ focal lens. The dimensions of the measurement volume were estimated to be $0.05 \mathrm{~mm} \times 0.7 \mathrm{~mm}$. Signals were processed by an IFA755 burst spectrum analyzer and velocity statistics were computed using the in-house ONERA software ASSA (Micheli et al. 2006). A total number of 300,000 samples was acquired for each measurement point. The measurements were performed along the test-cell centerline (at $y=0$ and $z=0$ ) as illustrated in Fig. 7.

Auto and cross power spectra estimates employed for the acoustic velocity components estimation method are evaluated using an interpolation method, re-sampling equidistantly the randomly sampled data at a constant frequency of $f_{s}=15 \mathrm{kHz}$. The effective number of points then used was usually about 250,000 as indicated in Tab. 2, which also provides the theoretical uncertainty of the estimation method with this number of samples. Bias associated with this interpolation approach was corrected at the acoustic frequency of interest $f_{a}$ using a synthetic tonal reference signal and invoking Parseval's theorem (Minotti et al. 2008). The results then obtained at the frequency of study are equal to the ones yielded by a slotting technique (Nobach 2002) with a relative difference usually lower than $1 \%$ and at a lower computational cost. While this LDV data post-processing method is by no means the most advanced one (see for example Buchhave and Velte (2015)) and might not account for all sources of bias induced by the LDV technique, it was found to be sufficient for the present purpose. 


\subsection{High-repetition-rate Particle Image Velocimetry}

The laser of the high-repetition-rate PIV system employed was a dual-cavity Litron LDY-304 laser, operated at a frequency $f_{s}=3 \mathrm{kHz}$. The laser, emitting at a wavelength $\lambda=$ $527 \mathrm{~nm}$, provides about $10 \mathrm{~mJ}$ per pulse at this repetition rate. Particle image pairs were acquired using a Phantom v711 camera, employed at a full resolution of $1280 \mathrm{px} \times$ 800 px. A $180 \mathrm{~mm}$ Sigma macro lens was mounted on the camera in order to visualize an area of $42 \mathrm{~mm} \times 25 \mathrm{~mm}$, that is $1.7 h \times 1.0 h$. The system is arranged in a $2 \mathrm{D} 2 \mathrm{C}$ configuration, measuring two velocity components in the $(x y)$ plane, at $z=0$ (see Fig. 7). Post-processing of the image pairs was performed with the in-house ONERA software FOLKISPIV (Champagnat et al. 2011), allowing a fast and accurate processing of the large amount of data acquired. Indeed, for each test case of Tab. 1, a minimum of 22,200 pairs of images were recorded in order to reach acceptable levels of uncertainty in the evaluation of the acoustic velocity components, as reported in Sec. 3.1. The number of PIV image pairs acquired for each test case is provided in Tab. 2 together with the theoretical statistical uncertainties to expect from Sec. 2. Note that the total number of image pairs that the camera can acquire at a time is limited to 11,100 with the current maximum 32GB internal memory. The total number of image pairs acquired for one case of study was then limited by the data transfer duration between the camera and the working station. An interrogation window with a size of 31 pixels was employed in the PIV post-processing, leading to a measurement spatial resolution of $1 \mathrm{~mm}$. The spatial scaling factor with this setup was $30 \mu \mathrm{m} / \mathrm{px}$.

Of particular importance was the laser interpulse time delay $\Delta t$, which was set to $15 \mu$ s based on the acoustic displacements associated with the lowest sound-pressure level studied. Indeed, for the case $L=130 \mathrm{~dB}$, the maximum acoustic displacement that can be observed is at best $\sqrt{2}\left|u_{\mathrm{rms}}^{\prime}\right|$ $=\left(0.23 \mathrm{~m} \mathrm{~s}^{-1}\right) \Delta t$ for small values of $\Delta t$ compared to the acoustic wave period. We can expect accurate PIV measurements with a correlation precision better than $0.1 \mathrm{px}$, that is $3 \mu \mathrm{m}$ given the spatial scaling factor obtained with this setup. The minimum value of $\Delta t$ then obtained is about $13 \mu \mathrm{s}$. For the higher sound pressure levels studied, there is no such constraint since the acoustic particle displacements are greater. Note that with this value of $\Delta t$, particle displacements of about 20 px are induced by the duct centerline mean velocity.

\section{Mean flow statistics}

The base flow in which acoustic excitations were introduced is first examined using the PIV measurements. Statistics were computed using 3,000 statistically independent instantaneous

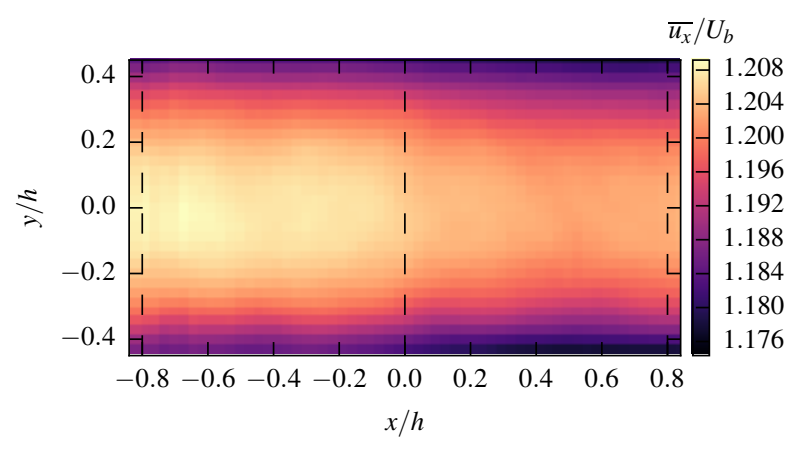

Fig. 8: Map of mean axial velocity $\overline{u_{x}}$ made dimensionless by the bulk velocity $U_{b}$ as measured by PIV in the plane $z=0$. Vertical dashed lines indicate the axial locations of the velocity profiles shown in Fig. 9.

velocity fields. A map of the dimensionless mean axial velocity $\overline{u_{x}} / U_{b}$ obtained is shown in Fig. 8. It can be observed that the maximum mean axial velocity is about $1.2 U_{b}$ on the centerline. Transverse mean axial velocity profiles extracted at three axial locations in the duct, indicated in Fig. 8 using dashed lines, are provided in Fig. 9. These mean axial velocity profiles have been made dimensionless by the duct centerline velocity $U_{c}$, showing a satisfactory overlap. It is emphasized that the apparent parabolic shape of these mean axial velocity profiles is only the result of a distortion caused by the scale of the figure axis and not by a laminar state of the flow, which is fully turbulent. The centerline velocity $U_{c}$, not shown here, slightly decreases streamwise, by a value of $0.2 \%$ of the bulk velocity $U_{b}$ in the measurement area: this decrease is low enough to consider the mean duct flow fully developed.

RMS axial and transverse velocity amplitudes, respec$\Delta t$ tively $\left(\overline{u_{x}^{\prime 2}}\right)^{1 / 2}$ and $\left(\overline{u_{y}^{\prime 2}}\right)^{1 / 2}$, made dimensionless by the bulk velocity $U_{b}$, are also presented in Fig. 9. As depicted, these turbulent quantities do not evolve streamwise, supporting that the turbulence of the duct flow is fully developed. The minimum value of $\left(\overline{u_{x}^{\prime 2}}\right)^{1 / 2}$ is obtained on the duct centerline and equals $4.4 \%$ of $U_{b}$. Large increases are observed symmetrically towards the walls. The RMS transverse velocity amplitudes $\left(\overline{u_{y}^{\prime 2}}\right)^{1 / 2}$ are lower, with values around $3.3 \%$ of $U_{b}$.

Power spectral density estimates $S_{u_{x} u_{x}} / U_{b}^{2}$ calculated from PIV and LDV measurements of the axial velocity $u_{x}$ at the center of the duct are displayed in Fig. 10. A satisfactory agreement is obtained between the two PSDs for frequencies lower than $10^{3} \mathrm{~Hz}$, thus for the energy-containing scales, supporting the validity of the two measurement setups and data post-processing. In this range, a fit proportional to the frequency $f$ to the power -0.65 yields a decent approximation of both PSDs. 


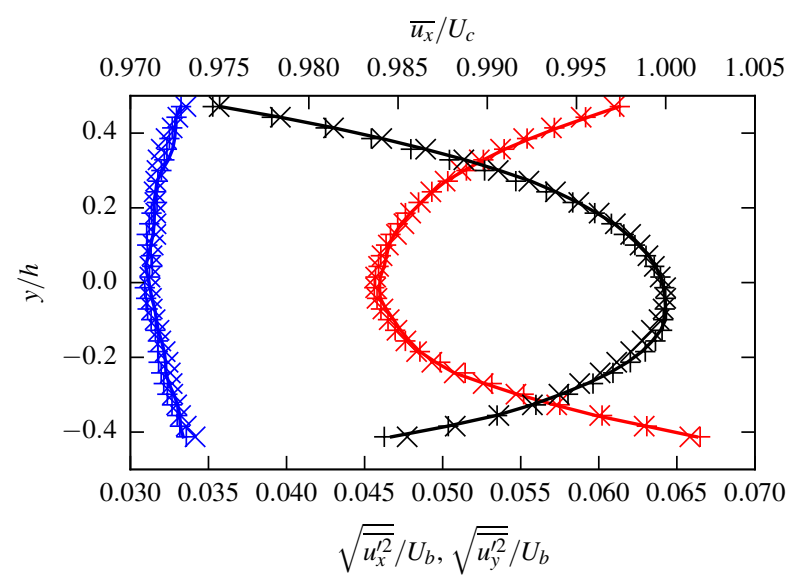

Fig. 9: Mean flow statistics as measured by PIV along the transverse direction $y$ at three axial locations $x$ : black line and symbols provide mean axial velocity measurements $\overline{u_{x}}$ made dimensionless by the centerline velocity $U_{c}$; red ones provide RMS axial velocity $\sqrt{\overline{u_{x}^{\prime 2}}}$ made dimensionless by the bulk velocity $U_{b}$; blue ones provide the dimensionless RMS transverse velocity $\sqrt{\overline{\overline{u_{y}^{\prime 2}}}} / U_{b}$. Lines $(-): x / h=0$; Cross symbols $(\times): x / h=-0.8$; Plus symbols $(+): x / h=$ 0.8 .

For the present setup, we can note that the high-repetitionrate PIV measurements cannot provide accurate spectral information above $1 \mathrm{kHz}$, where the inertial range is found. This range is however well resolved by LDV measurements up to a frequency of $7.5 \mathrm{kHz}$. In this region, a fitting exponent equal to -1.86 is obtained. This value is close to the classical $-5 / 3$ power-law that would be obtained assuming a Kolmogorov model together with Taylor's hypothesis to translate the law in the frequency domain, which is justified in the present case because of the large mean axial velocity compared to the turbulent velocity fluctuations. Finally, one can observe that the acoustic waves studied in the present work, that have a frequency $f_{a}=800 \mathrm{~Hz}$, are thus to be extracted from energy-containing scales. Studies that would be conducted at higher frequencies, would have to deal with scales containing much lower energy, yielding higher effective signal-to-noise ratios as discussed in Sec. 2.4.

\section{Acoustic velocity component estimations from laser-based measurements}

\subsection{Spatial filtering}

As explained in Sec. 3.1 using Tab. 2, the number of samples employed for PIV measurements could provide unsatisfactory uncertainty levels for the acoustic velocity com-

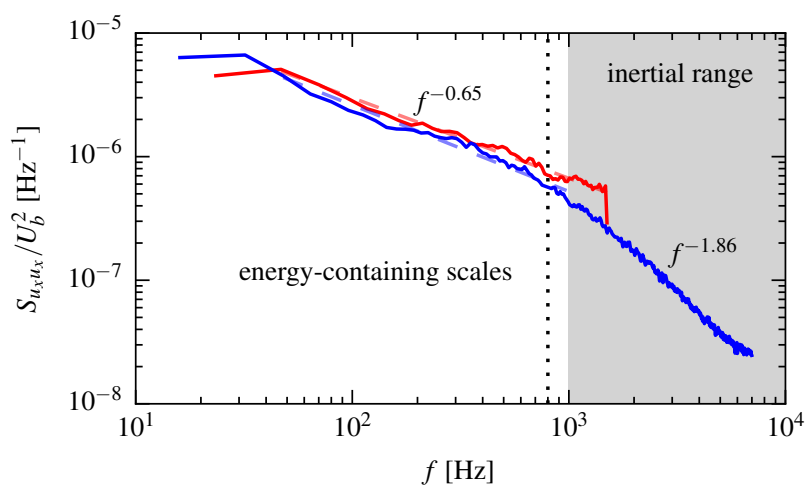

Fig. 10: Estimated power spectral densities of the PIV (—) and LDV (—) measurements of the axial velocity $u_{x}$ at the origin of the reference frame - i.e. center of the test cell; dashed straight lines represent local linear fits in log scales; the estimated levels of PSD at $f_{a}=800 \mathrm{~Hz}$ for PIV and LDV measurements are respectively $S_{\mathrm{PIV}}\left(f_{a}\right) / U_{b}^{2} \approx$ $6 \times 10^{-7} \mathrm{~Hz}^{-1}$ and $S_{\mathrm{LDV}}\left(f_{a}\right) / U_{b}^{2} \approx 5 \times 10^{-7} \mathrm{~Hz}^{-1}$.

ponent estimates. Since the estimation method as derived in Sec. 2.2 only relies on spectral characteristics of locally measured velocity components, spatial information may be used to improve the uncertainty of such estimates.

Indeed, for the present case of study where the walls of the test cell are rigid, it can easily be shown from Eq. (19) that the expected spatial wavenumber of the acoustic velocity amplitudes to be measured is given by

$k_{x}=\left|k^{+}-k^{-}\right| \approx 30 \mathrm{~m}^{-1}=0.75 h^{-1}$.

This wavelength does not vary with the sound pressure levels of the acoustic waves. Furthermore, the expected acoustic velocity phase differences to be measured are dominated by the downstream traveling wave, yielding an approximate phase difference spatial wavenumber of $k^{+} \approx 13 \mathrm{~m}^{-1}=0.33 \mathrm{~h}^{-1}$. Knowing these values, one can design an appropriate spatial filter that may be used to improve the acoustic velocity amplitude and phase difference estimates.

We investigated the application of a first-order low-pass 2D Gaussian filter on the maps of acoustic velocity amplitude and phase estimation calculated from PIV measurements, with a cut-off wavenumber $k_{c}=2.8 h^{-1}$, largely above the expected wavenumbers previously estimated, ensuring that no bias will be introduced in the estimates. This spatial filtering will only be applied in the following on the estimates yielded by the PIV measurements. It is emphasized that this approach is surely by no means the most efficient way to account for spatial coherence in order to improve the acoustic velocity components estimates but it is nonetheless a first step in this direction. 


\subsection{Confidence interval estimation using bootstrapping}

While one may rely on the analysis presented in Sec. 2.4 to estimate the uncertainty of the estimators considered, and thus provide some approximate confidence intervals, we aimed at supporting these results and providing more precise uncertainties through a more general, data-based approach that could also be applied to more complex cases. Indeed, for example, the previous uncertainty estimates obtained cannot account for further estimate refinements that would be provided taking into account spatial coherence, as proposed in Sec. 5.1. One way to address this issue is to rely on a bootstrap method (Efron and Tibshirani 1994).

Bootstrap methods provide a way to evaluate confidence intervals (CIs) associated with some sample estimate through random sampling with replacement. In our case, since the estimation approach detailed in Sec. 2.2 relies on a WOSA method, the sample that may be considered is the data set made of all the periodograms involved in this method, as briefly explained in Sec. 2.2. Indeed, the estimates are obtained by averaging these periodograms and taking the magnitude and phase of the result at the frequency of interest. One can easily add to these operations the spatial filtering previously defined, which would then be accounted for in the estimations of the CIs. Such an approach is more accurate in the determination of confidence intervals than just relying on the standard deviation of the estimators, which requires the assumption of a normal distribution of samples.

We performed such an analysis using the BCa method (Bias-Corrected Accelerated Non-Parametric) developed by Efron and Tibshirani (1994). In order to compare the results obtained using this data-based approach to the uncertainty estimates that can be evaluated using the analysis of Sec. 2.4, we calculated the 95\% CIs of the PIV- and LDVbased AVC estimates with a varying number of points $N$ and using $10^{4}$ bootstrap samples. Assuming a normal distribution of the AVC samples, an estimation of the uncertainties $\hat{\sigma}_{\hat{A}}$ and $\hat{\sigma}_{\hat{\phi}}$ can be obtained in an approximate manner relying on these results. This is performed by considering the two-sided confidence limits evaluated through bootstrapping, calculating their absolute difference to the estimate and dividing by 1.96 . Indeed, in the case of normally distributed samples, the confidence intervals defined by $\pm 1.96 \sigma$ provide a $95 \%$ probability to contain the true parameter values. The results of this procedure are gathered in Fig. 11 for the case of a SPL at $L=130.5 \mathrm{~dB}$ and at the test-cell center. Similar results were obtained for other SPLs and spatial locations. We emphasize that in the present results, no spatial filtering has yet been applied to the PIV measurements.

First, a clear convergence of the experimental uncertainty levels is obtained with an increasing number of samples for both PIV- and LDV-based estimates. Such a behavior was displayed in Fig. 3 using synthetic data and is here observed

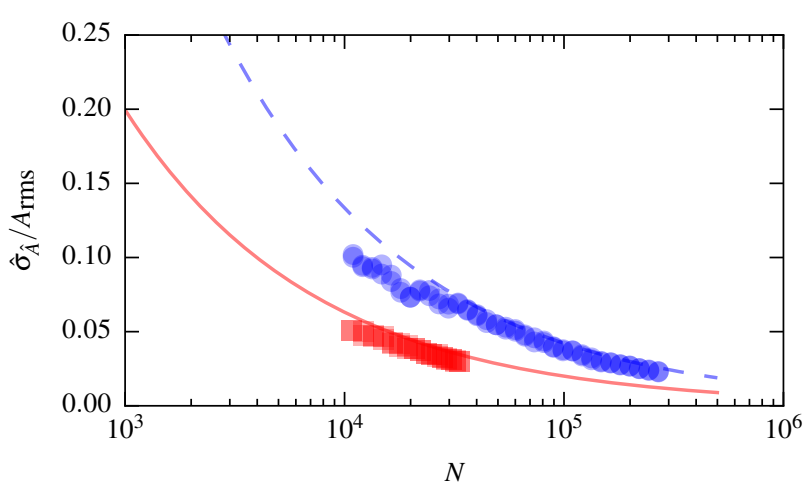

(a)

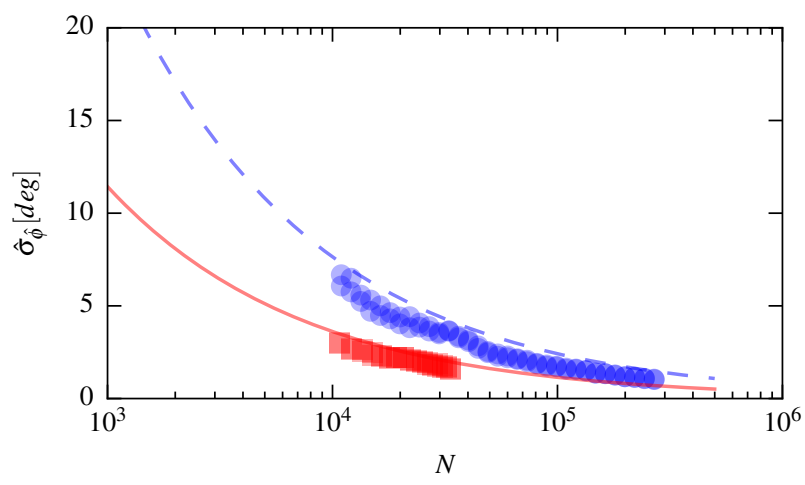

(b)

Fig. 11: Estimated experimental uncertainty of acoustic axial velocity RMS amplitude (a) and phase difference (b) yielded by the bootstrap method applied to the raw PIV ( $\square$ ) and LDV (O) data, as a function of the number of measurement points $N$ considered and assuming a normal distribution of the samples; results obtained at $x / h=0.0$ for $L=130.5 \mathrm{~dB}$; the solid $(-)$ and dashed $\left(-{ }_{--}\right)$lines are the CRLBs obtained from the inequalities (10) with respectively SNR $f=-16.1 \mathrm{~dB}(\mathrm{PIV})$ and $\mathrm{SNR}_{f}=-22.5 \mathrm{~dB}$ (LDV), as indicated in Tab. 1.

with measurements, bringing further confidence in the present results which appear to asymptotically converge.

Second, in this figure are also displayed the theoretical uncertainties evaluated using the CRLBs defined by the inequalities (10) and relying on the frequency-dependent $\mathrm{SNR}_{f}$ provided in Tab. 1 rather than the global SNR. Clearly, even if the approximate experimental uncertainties do not perfectly collapse on these theoretical curves, the trends are satisfactorily reproduced, supporting the analysis performed in Sec. 2.4. Particularly, one can observe a clear difference between PIV and LDV estimate uncertainties, which is reproduced by using the present values of $\mathrm{SNR}_{f}$.

This bootstrap method was then applied to the entire set of PIV and LDV measurements, with the maximum number 


\begin{tabular}{|c||c|c||c|c|}
\hline \multicolumn{1}{|c||}{} & \multicolumn{2}{c||}{ PIV } & \multicolumn{2}{c|}{ LDV } \\
\hline$L[\mathrm{~dB}]$ & $\hat{\sigma}_{\hat{A}} / A_{\mathrm{rms}}$ & $\hat{\sigma}_{\hat{\phi}}$ & $\hat{\sigma}_{\hat{A}} / A_{\mathrm{rms}}$ & $\hat{\sigma}_{\hat{\phi}}$ \\
\hline 130.5 & $1.9 \%$ & $1.3^{\circ}$ & $1.7 \%$ & $1.0^{\circ}$ \\
138 & $1.1 \%$ & $0.9^{\circ}$ & $0.7 \%$ & $0.4^{\circ}$ \\
145 & $0.6 \%$ & $0.5^{\circ}$ & $0.5 \%$ & $0.2^{\circ}$ \\
150 & $0.4 \%$ & $0.2^{\circ}$ & $0.5 \%$ & $0.1^{\circ}$ \\
\hline
\end{tabular}

Table 3: Estimated mean experimental uncertainty levels of the acoustic velocity component estimates deduced from the bootstrap method applied on the PIV and the LDV measurements, assuming a normal distribution of the samples.

of samples $N$ available for each case. Spatial filtering was here activated for the processing of the PIV data. The mean estimated uncertainty levels of the AVC estimates obtained following the previous approach are gathered in Tab. 3. These uncertainties are in decent agreement with the theoretical ones presented in Tab. 2, especially for LDV measurements since no spatial filtering was performed on these estimations.

\subsection{Acoustic velocity component estimations}

AVC estimation results, together with the 95\% CIs evaluated through bootstrapping, are gathered in the four figures Fig. 12, Fig. 13, Fig. 14 and Fig. 15. These figures are respectively displaying the acoustic velocity amplitude and phase difference estimates obtained for $L=130.5 \mathrm{~dB}$, $L=138 \mathrm{~dB}, L=145 \mathrm{~dB}$ and $L=150 \mathrm{~dB}$. Only the results obtained for the axial acoustic velocity component are presented, the transverse component being negligible. Maps yielded by PIV data and more quantitative plots of acoustic velocity amplitude estimates are provided in figures (a), while phase difference estimates are shown in figures (b).

First of all, one can observe in the map of Fig. 12a that acoustic velocity amplitude estimates obtained with the PIV data are globally scattered between $0.14 \mathrm{~m} \mathrm{~s}^{-1}$ and $0.16 \mathrm{~m} \mathrm{~s}^{-1}$, which is consistent with the theoretical value provided in Tab. 1 for the case of a SPL at $130.5 \mathrm{~dB}$. The values of these PIV-based estimates obtained on the duct axis $y=0$ are reported in the lower plot and displayed with a solid blue line. With this line are associated the $95 \%$ CIs as evaluated using the bootstrap method of Sec. 5.2 and displayed using a semitransparent blue area. For completeness only, the raw PIVbased estimates are also depicted, using a solid light-blue line. These PIV-based estimates are to be compared with the LDV-based estimates (circle symbols with error bars showing the bootstrap $95 \% \mathrm{CI}$ ), the theoretical trends yielded by Eq. (19) (dashed black line) and the measurements obtained using wall-microphones (red square symbols with gray error bars). A very similar representation is provided in Fig. 12b, giving the axial evolution of the measured phase difference estimates. The plots shown in figures Fig. 13, Fig. 14 and

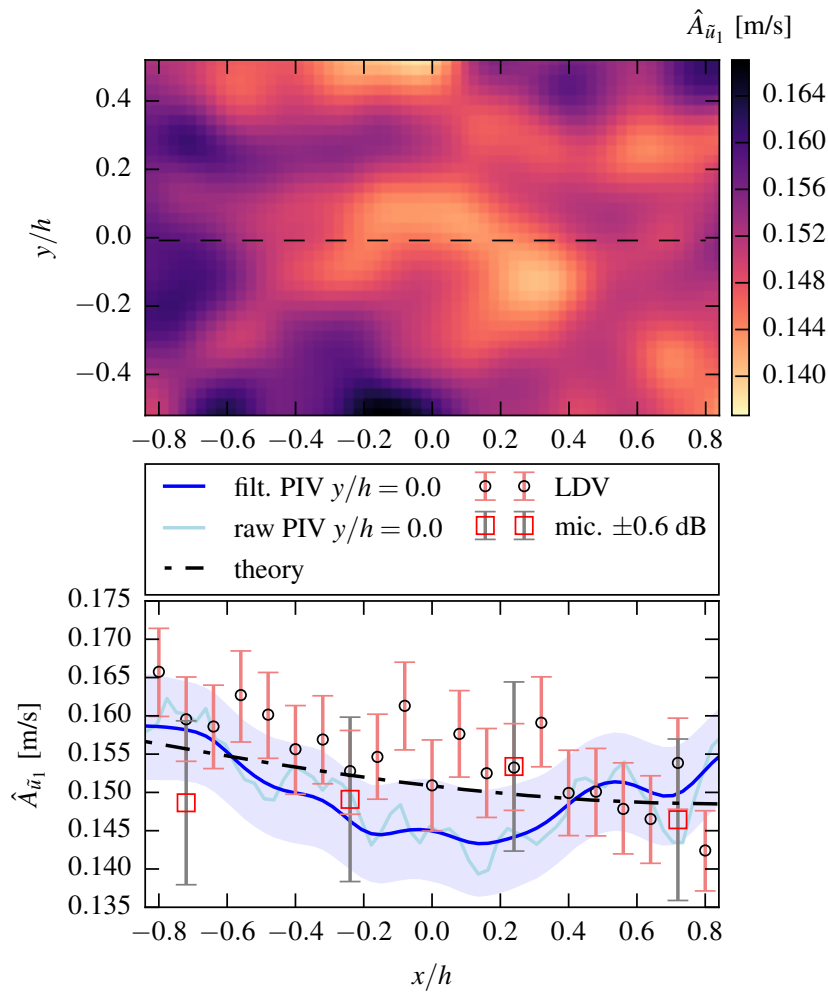

(a)

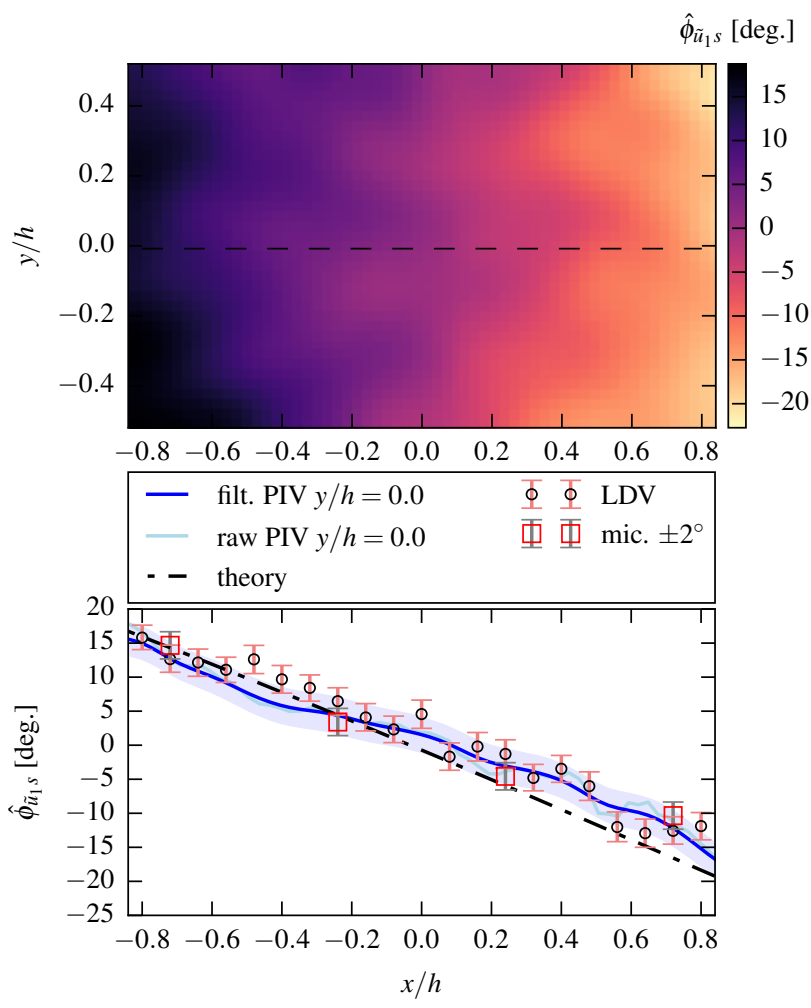

(b)

Fig. 12: Acoustic velocity amplitude and phase estimates at $L=130.5 \mathrm{~dB}$. 


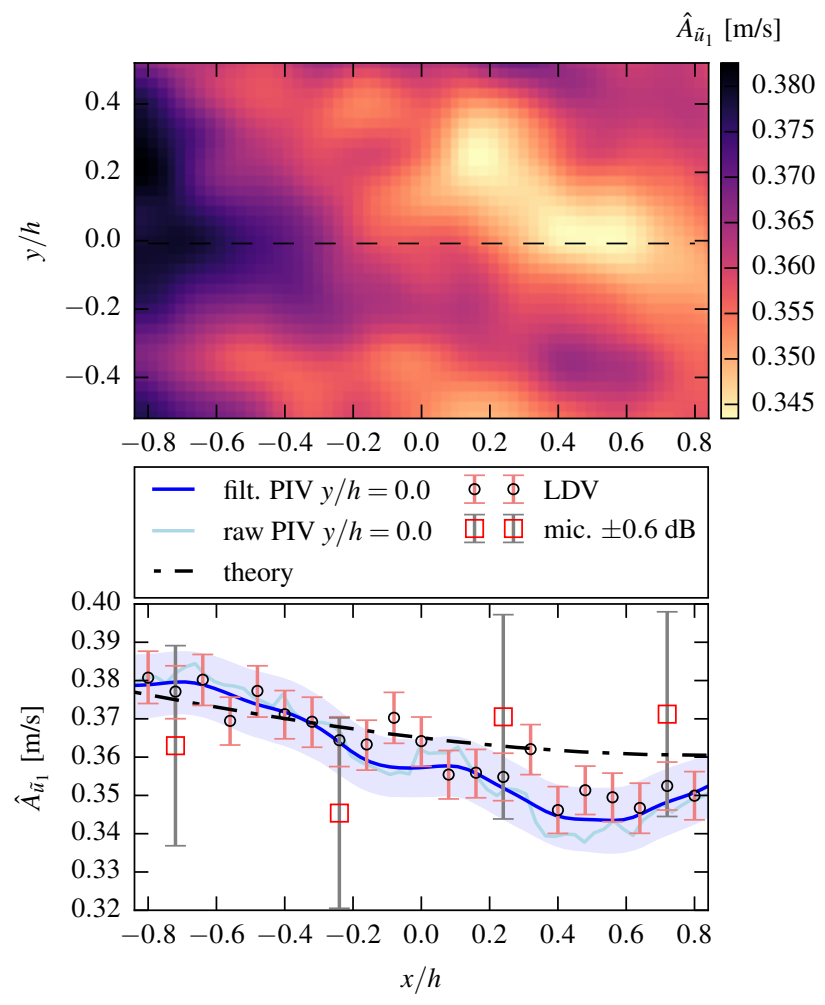

(a)

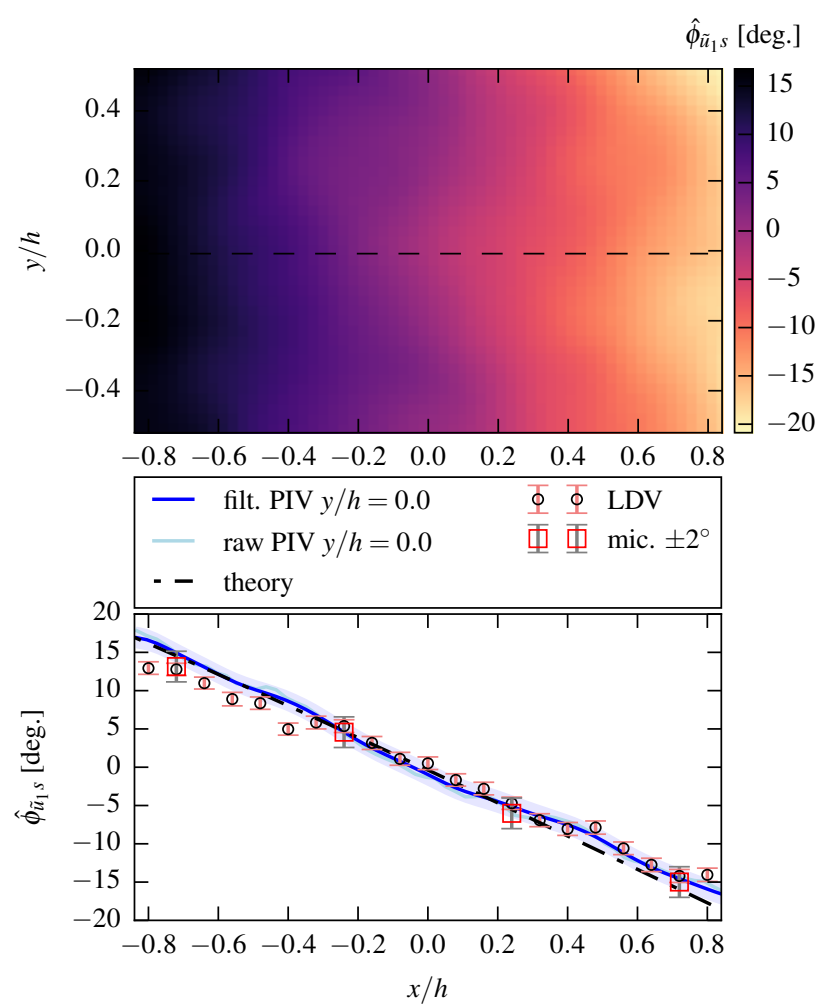

(b)

Fig. 13: Acoustic velocity amplitude and phase estimates at $L=138 \mathrm{~dB}$.

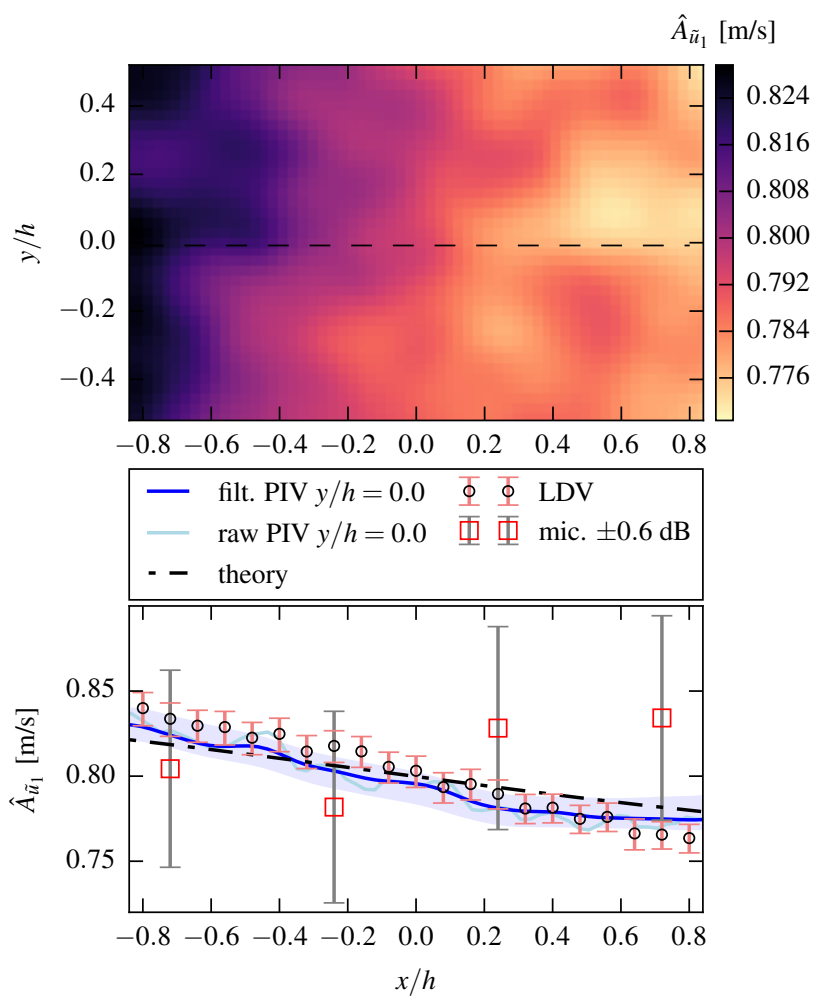

(a)

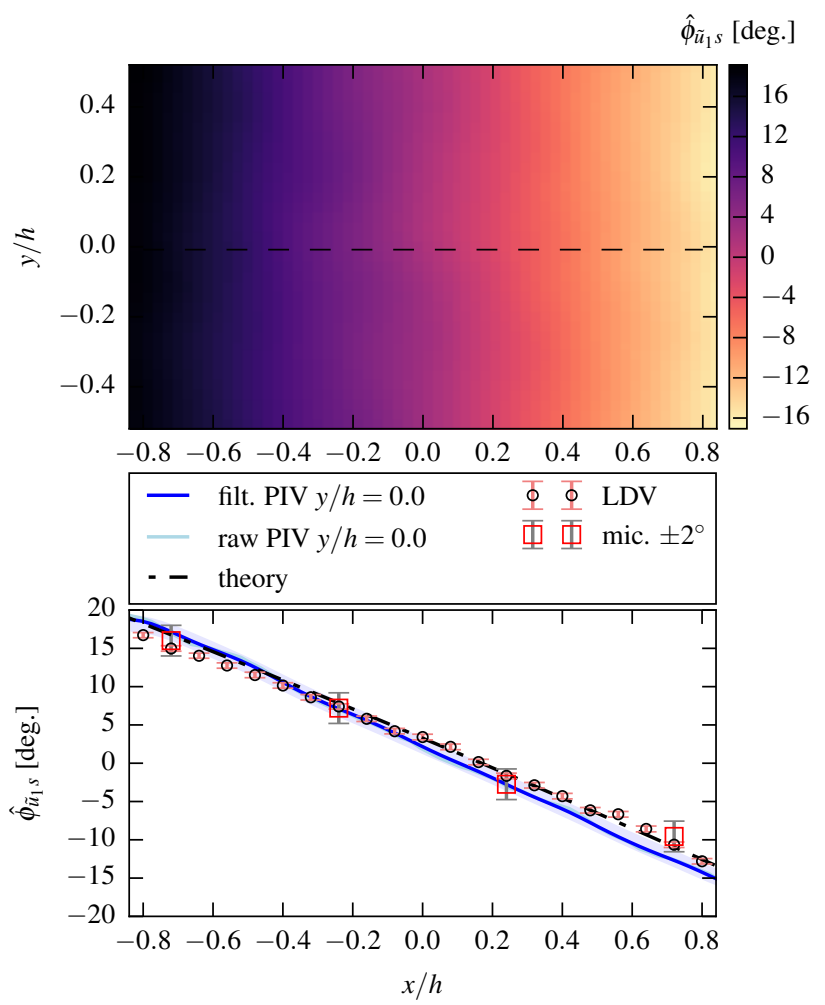

(b)

Fig. 14: Acoustic velocity amplitude and phase estimates at $L=145 \mathrm{~dB}$. 


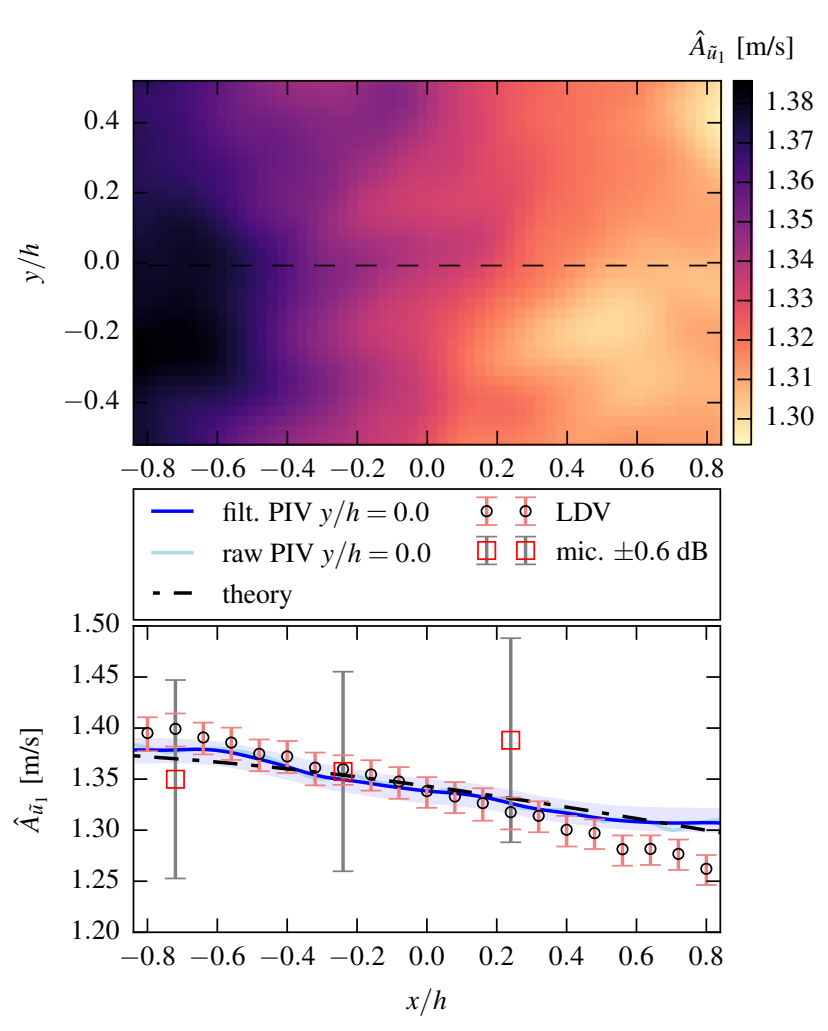

(a)

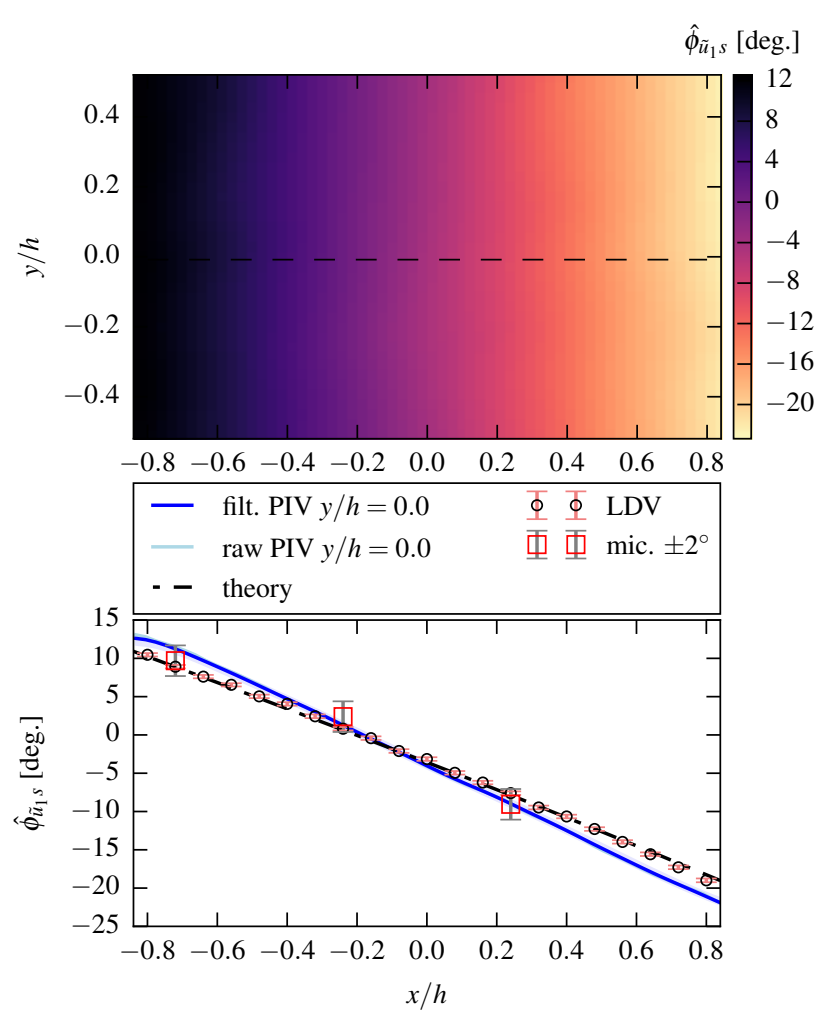

(b)

Fig. 15: Acoustic velocity amplitude and phase estimates at $L=150 \mathrm{~dB}$.
Fig. 15 provide in a similar manner the results obtained for the higher SPL.

Overall, we consider that the various measurement techniques employed yield very consistent results on both acoustic velocity amplitude and phase difference estimations. PIVbased and LDV-based estimates seem to present in some cases slight discrepancies in terms of magnitude or phase slope, especially for the highest SPL of $150 \mathrm{~dB}$. But considering the $95 \%$ CIs and knowing that the two sets of measurements were performed with a time interval of several months, implying slightly different experimental conditions especially on the acoustic wave levels due to a high sensitivity of the loudpseakers command system, we consider this cross-validation as satisfactory. These laser-based estimates are furthermore satisfactorily following the decreasing trends of the theoretical acoustic amplitude curves that decrease on the measurement area considered. Phase difference trends are globally very well recovered, except for the PIV-based results at the highest SPL, which may be explained by the previous arguments.

Finally, we can consider that the values provided by the wall-pressure measurements are also in decent agreement with the laser-based results. Indeed, firstly, as emphasized previously, these estimations rely on the very simplifying assumption of a uniform mean flow. Secondly, such measurements are considered to present an uncertainty in terms of sound pressure levels of about $\pm 0.3 \mathrm{~dB}$ and thus approximate $95 \%$ confidence intervals of $\pm 0.6 \mathrm{~dB}$, as reported in these plots using error bars. These CIs expressed in decibels are then converted in CIs expressed in $\mathrm{m} \mathrm{s}^{-1}$ using Eq. (14) and Eq. (15). For the phase measurements, we consider a $95 \%$ CI given by $\pm 2^{\circ}$. Clearly, the laser-based measurement uncertainties are well within the uncertainty levels generally admitted for microphone-based measurements for every case of study, supporting the validity and the relevance of the measurement approaches presented. We note that, leaving aside the mean flow assumption issue that would affect the estimation trueness, more precise microphone-based estimates may be obtained by repeating the measurement several time, taking then into account systematic and operatordependent bias, or by improving the flush-mounted microphone setup.

\section{Conclusions}

A method was presented for estimating acoustic velocity components, that is amplitude and phase difference, of multisine acoustic waves carried in a fully turbulent flow. This approach relies on the estimation of cross-power spectra between velocity measurements and a reference signal correlated with the acoustic waves studied. While such an approach has already been successfully employed by Minotti 
et al. (2008) for example, no theoretical details or analysis regarding its efficiency is available in the literature.

The present study provides supporting evidence that the acoustic velocity amplitude and phase difference estimators built using this approach are asymptotically efficient with an increasing number of samples when considering White Gaussian Noise (WGN). This result was obtained by comparing the variance of the estimators with the corresponding Cramér-Rao lower bounds on synthetic cases. The analysis conducted also provides theoretical lower bounds for the estimators uncertainties. Furthermore, it was emphasized that application cases for which this estimation approach has been developed, that is acoustic waves in turbulent duct flows, bring into play turbulence spectra that are not well modeled using WGN, owing to the non-uniformity of power spectral density distributions classically encountered in such flows. Consequently, a more representative description of the estimator uncertainty lower bounds was proposed, taking into account such a spectral feature and rendering the uncertainty estimates frequency-dependent. Particularly, the higher the acoustic wave frequency, the lower the theoretical estimators uncertainties.

In order to confirm the validity of both the AVC estimation method studied and the uncertainty estimation approach proposed, measurements were performed in a fully turbulent duct flow with a bulk Mach number $M_{b}=0.1$. For the sake of simplicity only, single-tone acoustic waves were added to the flow, with sound pressure levels above $130 \mathrm{~dB}$ giving overall signal-to-noise ratios above $-19 \mathrm{~dB}$. Two laser-based measurement campaigns were conducted, using LDV and high-repetition-rate PIV to measure velocity fluctuations in the center of the duct. In addition, wall pressure measurements were acquired for reference. It was shown that consistent acoustic velocity component estimates were obtained for both laser-based techniques and for all cases of study. Furthermore, comparing the results with microphone measurements, it is reported that the laser-based measurement uncertainties for the present cases of study are largely within the usual range of uncertainties accepted using microphones, in terms of sound pressure levels. Finally, the experimental uncertainties, evaluated using a bootstrap method on the periodograms employed in the estimation process, are in fair agreement with the ones derived using a simple frequency-dependent model which may thus be employed for design purpose.

As stated in the article, the acoustic velocity component estimation approach here presented is directly applicable to multi-sine acoustic wave, contrary to phase-averaged techniques. Future work will then focus on its application to study acoustic liner responses in duct flows with multisine acoustic forcing, using both high-repetition rate PIV and LDV measurements. Finally, the methods presented in this work should also be suitable for the non-intrusive study of aeroacoustic sound sources in wind-tunnels. Indeed, provided that a suitable reference signal such as a microphone measurement is available, acoustic velocity fields generated by a sound source can be accurately evaluated in the regions of the flow where the turbulence is not correlated with the acoustic velocity components. Future works will investigate such applications.

Acknowledgements This work was partially performed in the framework of the ONERA intern research project 3DFlow and has been partially funded within the frame of the Joint Technology Initiative JTI Clean Sky 2, Large Passenger Aircraft Innovative Aircraft Demonstration Platform "LPA IADP" platform (contract $\mathrm{N}^{\circ}$ CSJU-CS2-GAMLPA-2014-2015-01) being part of the Horizon 2020 research and innovation framework programme of the European Commission. Nicolas Fasano is warmly acknowledged for his machining experience and helping with the experimental setup. The authors thank Francis Micheli for his work on the LDV system.

\section{References}

Alomar A, Aurégan Y (2017) Particle image velocimetry measurement of an instability wave over a porous wall in a duct with flow. Journal of Sound and Vibration 386:208-224

Betgen B, Galland MA, Piot E, Simon F (2012) Implementation and non-intrusive characterization of a hybrid activepassive liner with grazing flow. Applied Acoustics 73(6-7):624-638, DOI 10.1016/ j.apacoust.2012.01.008, URL http://linkinghub.elsevier. com/retrieve/pii/S0003682X12000254

Bodén H (2013) Acoustic properties of perforates under high level multi-tone excitation. In: 19th AIAA/CEAS Aeroacoustics Conference, p 2175, DOI http://dx.doi.org/10.2514/6.2013-2175

Bodén H, Abom M (1986) Influence of errors on the twomicrophone method for measuring acoustic properties in ducts. The Journal of the Acoustical Society of America 79(2):541-549, DOI http://dx. doi.org/10.1121/1.393542, URL http://scitation.aip.org/ content/asa/journal/jasa/79/2/10.1121/1.393542

Breakey DES, Fitzpatrick JA, Meskell C (2013) Aeroacoustic source analysis using time-resolved piv in a free jet. Experiments in Fluids 54(5):1531, DOI 10.1007/s00348-013-1531-5, URL http: //dx.doi.org/10.1007/s00348-013-1531-5

Buchhave P, Velte CM (2015) Reduction of noise and bias in randomly sampled power spectra. Experiments in Fluids 56(4):79, DOI 10.1007/s00348-015-1922-x, URL http://dx.doi.org/ $10.1007 / \mathrm{s} 00348-015-1922-\mathrm{x}$

Champagnat F, Plyer A, Le Besnerais G, Leclaire B, Davoust S, Le Sant Y (2011) Fast and accurate piv computation using highly parallel iterative correlation maximization. Experiments in Fluids 50(4):1169-1182, DOI 10.1007/s00348-011-1054-x, URL http: //dx.doi.org/10.1007/s00348-011-1054-x

Chung JY (1977) Rejection of flow noise using a coherence function method. The Journal of the Acoustical Society of America 62(2):388, DOI 10.1121/1.381537, URL http://scitation.aip.org/content/asa/journal/ jasa/62/2/10.1121/1.381537

Chung JY, Blaser DA (1980) Transfer function method of measuring acoustic intensity in a duct system with flow. Acoustical Society of America Journal 68:1570-1577, DOI 10.1121/1.385211

Efron B, Tibshirani R (1994) An Introduction to the Bootstrap. Chapman \& Hall/CRC Monographs on Statistics \& Applied Probability, Taylor \& Francis

Fischer A, Büttner L, Czarske J, Eggert M, Grosche G, Mller $\mathrm{H}$ (2007) Investigation of time-resolved single detector 
Doppler global velocimetry using sinusoidal laser frequency modulation. Measurement Science and Technology 18(8):2529-2545, DOI 10.1088/0957-0233/18/8/029, URL http: //stacks. iop.org/0957-0233/18/i=8/a=029?key= crossref.cff513934aa1ef8d38d21fba5592f 264

Fischer A, Sauvage E, Roehle I (2008) Acoustic PIV: Measurement of the acoustic particle velocity using synchronized PIV-technique. The Journal of the Acoustical Society of America 123(5):3130, DOI 10.1121/1.2933079, URL http://scitation.aip.org/ content/asa/journal/jasa/123/5/10.1121/1.2933079

Fischer A, König J, Haufe D, Schlüßler R, Büttner L, Czarske J (2013) Optical multi-point measurements of the acoustic particle velocity with frequency modulated Doppler global velocimetry. Acoustical Society of America Journal 134:1102, DOI 10.1121/1.4812753

Haufe D, Schlüßler R, Fischer A, Büttner L, Czarske J (2012) Optical multi-point measurement of the acoustic particle velocity in a superposed flow using a spectroscopic laser technique. Measurement Science and Technology 23(8):085,306, DOI 10.1088/0957-0233/23/8/085306, URL http: //stacks.iop.org/0957-0233/23/i=8/a=085306? key=crossref . 66c392a728e04401b441da1c6ca8ef 1e

Haufe D, Fischer A, Czarske J, Schulz A, Bake F, Enghardt L (2013) Multi-scale measurement of acoustic particle velocity and flow velocity for liner investigations. Experiments in Fluids 54(7), DOI 10.1007/s00348-013-1569-4, URL http://link. springer .com/10.1007/s00348-013-1569-4

Heinzel G, Rüdiger A, Schilling R (2002) Spectrum and spectral density estimation by the Discrete Fourier transform (DFT), including a comprehensive list of window functions and some new at-top windows

Henning A, Koop L, Ehrenfried K (2010) Simultaneous Particle Image Velocimetry and Microphone Array Measurements on a Rod-Airfoil Configuration. AIAA Journal 48:2263-2273, DOI 10.2514/1.J050314

Henning A, Kröber S, Koop L (2013) Acoustic particle velocity measurements: a cross comparison between modern sensor technologies. Measurement Science and Technology 24(8):085,303, URL http://stacks. iop. org/0957-0233/24/i=8/a=085303

Heuwinkel C, Fischer A, Röhle I, Enghardt L, Bake F, Piot E, Micheli F (2010) Characterization of a perforated liner by acoustic and optical measurements. vol 16th AIAA/CEAS Aeroacoustics Conference (31st AIAA Aeroacoustics Conference)

Kay S (1984) Fundamentals of Statistical Signal Processing, Volume 1: Estimation Theory. Pearson Education

Micheli F, Lavieille M, Millan P (2006) Assa, un outil de référence pour le traitement du signal en vélocimétrie laser. In: 10e Congrès Francophone de Techniques Laser (CFTL), Toulouse (France)

Minotti A, Simon F, Gantié F (2008) Characterization of an acoustic liner by means of Laser Doppler Velocimetry in a subsonic flow. Aerospace Science and Technology 12(5):398-407, DOI 10.1016/ j.ast.2007.09.007, URL http://linkinghub.elsevier.com/ retrieve/pii/S1270963807001150

Nobach H (2002) Local time estimation for the slotted correlation function of randomly sampled lda data. Experiments in Fluids 32(3):337-345, DOI 10.1007/s003480100362, URL http: //dx . doi.org/10.1007/s003480100362

Piot E, Micheli F, Simon F (2010) Optical acoustic pressure measurements in a large-scale test facility with mean flow. vol 16th AIAA/CEAS Aeroacoustics Conference (31st AIAA Aeroacoustics Conference)

Rausch A, Fischer A, Kings N, Bake F, Roehle I (2012) Optical measurement of acoustic pressure amplitudesat the sensitivity limits of Rayleigh scattering. Optics Letters 37(13):2685, DOI 10.1364/ OL.37.002685, URL https://www.osapublishing.org/ol/ abstract. cfm?uri=ol-37-13-2685
Stoica P, Moses RL, et al. (2005) Spectral analysis of signals, vol 452. Pearson Prentice Hall Upper Saddle River, NJ

Valière JC (2014) Acoustic Particle Velocity Measurements Using Laser: Principles, Signal Processing and Applications. FOCUS Series, Wiley 